Pacific

Journal of

Mathematics

\title{
STRONG ADDITIVITY AND CONFORMAL NETS
}

FENG XU 


\title{
STRONG ADDITIVITY AND CONFORMAL NETS
}

\author{
FENG XU \\ Dedicated to Masamichi Takesaki on his 70th birthday
}

\begin{abstract}
We show that the fixed-point subnet of a strongly additive conformal net under the action of a compact group is strongly additive. Using the idea of the proof we define the notion of strong additivity for a pair of conformal nets and we show that a key fact about induction of pairs, proved earlier under the assumption of finite index, can be generalized to strongly additive pairs of conformal nets. These results are used to classify conformal nets of central charge $c=1$ that are not necessarily rational and satisfy a spectrum condition.
\end{abstract}

\section{Introduction}

We use the letters $I$ and $J$ to denote intervals, by which we mean nonempty open connected subsets of the circle whose complement has nonempty interior. A conformal net is a map $A: I \mapsto \mathscr{A}(I)$ from the set of intervals to the set of von Neumann algebras on a fixed separable Hilbert space, subject to certain compatibility conditions spelled out on page 170. It is proved in [Fredenhagen and Jörß 1996] that any conformal net $\mathscr{A}$ is additive, in the sense that if $I_{n}$ is a sequence of intervals that cover an interval $J$, then $\bigvee_{n} \mathscr{A}\left(I_{n}\right)$ contains $\mathscr{A}(J)$, where $\bigvee_{n} \mathscr{A}\left(I_{n}\right)$ is the von Neumann algebra generated by the $\mathscr{A}\left(I_{n}\right)$, for $n \in \mathbb{N}$. We say that $\mathscr{A}$ is strongly additive if $\mathscr{A}\left(I_{1}\right) \vee \mathscr{A}\left(I_{2}\right)=\mathscr{A}(I)$ whenever $I, I_{1}, I_{2}$ are intervals such that $I_{1} \cup I_{2}$ is obtained from $I$ by removing an interior point. A conformal net that violates strong additivity is given in [Buchholz and Schulz-Mirbach 1990]. Strong additivity seems like a rather technical condition, but it plays an important role in the study of representations of conformal nets [Kawahigashi et al. 2001; Xu 2001].

In [Xu 2001, Proposition 2.8] we proved that the fixed-point subnet $\mathscr{B}$ (see Section 3 ) of a strongly additive net $\mathscr{A}$ under the action of a finite group is strongly additive. The proof used the Galois correspondence for the action of the finite group on von Neumann algebras. This result was generalized in [Longo 2003] to the case when $\mathscr{B} \subset \mathscr{A}$ has finite Jones index, using the notion of transplanting

MSC1991: 46S99, 81R10.

Keywords: subfactor, conformal net, strong additivity.

This work is partially supported by an NSF grant. 
conformal subnets. Both proofs depend on the finite-index condition and it is not obvious how to generalize them to the case when the finite group is replaced by a compact but infinite group.

Theorem 2.6 in this article shows that the result cited is still true when instead of a finite group we use a compact group. We use the results of [Haagerup 1978], and the idea, as in [Xu 2001], is to show that

$$
\mathscr{A}\left(I_{1}\right) \vee \mathscr{B}\left(I_{2}\right)=\mathscr{A}(I),
$$

where $I_{1}, I_{2}, I$ are as above and we use a dilation argument similar to the one in [Xu 2001]. But the method of proof differs from [Xu 2001] and [Longo 2003], and it leads to a simpler proof of the strong additivity results in those two works (see Remark 2.7).

In Section 3 we consider a pair $\mathscr{B} \subset \mathscr{A}$ of conformal nets and extend results of [Böckenhauer and Evans 1998; 1999b; 1999a; Longo and Rehren 1995; Xu 1998] to the present setting. Proposition 3.1 is essentially found in [Longo and Rehren 1995], except that with additivity we can get stronger properties, as proved in [Xu 1998] for the finite-index case. The next several results are also extensions to the current case of results proved in [Xu 1998] under the finite-index condition.

It turns out that (1-1) can be used to generalize [Xu 1998, Theorem 3.3], concerning the induction of the pair $\mathscr{B} \subset A$, which is of interest since such results have found many applications [Böckenhauer and Evans 1998; 1999b; 1999a; Fuchs et al. 2002; Petkova and Zuber 2002]. We thus define a pair of nets $\mathscr{B} \subset \mathscr{A}$ to be strongly additive if it satisfies (1-1); many examples of such pairs arise from Lemma 3.6 and Proposition 3.7. The generalization of [Xu 1998, Theorem 3.3] to strongly additive pairs is Theorem 3.8, which together with its corollary provide powerful tools for the study of induced endomorphisms, as shown in [Xu 1998] for the finiteindex case. (Other results in [Xu 1998] also generalize to our current setting and we plan to return to them in the future.)

In Section 4 we apply these results to classify conformal nets $\mathscr{A}$ with central charge $c=1$. The idea is simple and goes roughly as follows. The $c=1$ Virasoro subnet $\mathscr{B} \subset \mathscr{A}$ has a representation - the vector representation - with Jones index 4 (Lemma 4.1), and since $\mathscr{B}$ is strongly additive by Theorem 2.6 , the induced endomorphism to $\mathscr{A}$ has Jones index 4 by Proposition 3.4 and Lemma 4.1. Hence the principal graph of this induced endomorphism is one of the A-D-E graphs listed in [Goodman et al. 1989], and in fact such subfactors are classified in [Popa 1995]. Depending on the nature of the principal graph, we can obtain enough information to identify $\mathscr{A}$, if we assume a Spectrum Condition (page 191) to the effect that any degenerate representation of the Virasoro net other than the vacuum representation must appear in $\mathscr{A}$ if $\mathscr{A} \neq \mathscr{B}$. This condition is true for all known examples and we conjecture that it is always true. 
An A-D-E type classification for $c<1$ was given in [Kawahigashi and Longo 2004], and there are some similarities between the results in that article and ours. But there are notable differences, since for $c=1$ the Virasoro net is not rational, and the results of Sections 2 and 3 play a crucial role in our proofs. We have tried to give different proofs for each case in that discussion, since we expect that the ideas of these proofs, as well as the general results of Sections 2 and 3, will have applications beyond those described in Section 4.

After this paper appeared on the web, we were informed by Professor Roberto Longo that the first two cases of our analysis in the proof of Theorem 4.6 overlap with unpublished results of S. Carpi; see [Kawahigashi 2003].

\section{Strong additivity of subnets}

Sectors. Let $M$ be a properly infinite factor and End $M$ the semigroup of unitpreserving endomorphisms of $M$. In this paper $M$ will always be of the unique hyperfinite $\mathrm{III}_{1}$ type. Taking the quotient of End $M$ modulo unitary equivalence in $M$ we get the semiring Sect $M$ of sectors of $M$, which is endowed with a natural involution $\theta \rightarrow \bar{\theta}$ [Longo 1989; 1990].

Given $\rho \in$ End $M$ and a normal faithful conditional expectation $\epsilon: M \rightarrow \rho(M)$, we define $d_{\epsilon} \in(0,+\infty]$ by

$$
d_{\epsilon}^{-2}:=\max \left\{\lambda \in[0,+\infty) \mid \epsilon(m) \geq \lambda m \quad \text { for all } m \in M_{+}\right\}
$$

[Pimsner and Popa 1986]. We also define $d_{\rho}=\min _{\epsilon}\left\{d_{\epsilon}\right\}$ and call this number the statistical dimension of $\rho$; its square $d_{\rho}^{2}$ is called the (minimal) index of $\rho$. Clearly $d_{\rho}$ depends only on the unitary equivalence class $[\rho]$ of $\rho$. For the properties of $d_{\rho}$ see [Longo 1989; 1990; 1992].

For $\lambda, \mu \in$ End $M$, let $\operatorname{Hom}(\lambda, \mu)$ denote the space of intertwiners from $\lambda$ to $\mu$, that is, elements $a \in M$ such that $a \lambda(x)=\mu(x) a$ for any $x \in M$. The dimension of the vector space $\operatorname{Hom}(\lambda, \mu)$ is denoted by $\langle\lambda, \mu\rangle$; it depends only on $[\lambda]$ and $[\mu]$. If $\nu, \lambda$ and $\mu$ have finite index, Frobenius duality gives $\langle\nu \lambda, \mu\rangle=\langle\lambda, \bar{v} \mu\rangle$ and $\langle v \lambda, \mu\rangle=\langle\nu, \mu \bar{\lambda}\rangle$ [Longo 1994]. If $\mu$ is a subsector (direct summand) of $\lambda$, we write $\mu \prec \lambda$ or $\lambda \succ \mu$. A sector is said to be irreducible if it has only one subsector. We will sometimes use 1 to denote the identity sector if no confusion is possible.

If $\lambda$ is a sector with finite statistical dimension, the principal graph $\Gamma$ of $\lambda$ is a bipartite graph defined as follows. The even vertices of $\Gamma$, denoted by $\Gamma_{0}$, are labeled by the irreducible sectors of $(\bar{\lambda} \lambda)^{n}, n \in \mathbb{N}$, and the odd vertices of $\Gamma_{1}$ of $\Gamma$ are labeled by the irreducible sectors of $(\bar{\lambda} \lambda)^{n} \lambda, n \in \mathbb{N}$. An even vertex $x$ is connected to an odd vertex $y$ by $\langle x \lambda, y\rangle$ edges. We say that $\lambda$ has finite depth if $\Gamma$ is a finite graph. We say that $\lambda$ is amenable if $\|\Gamma\|=d_{\lambda}$, where $\Gamma$ is considered a linear map from $l^{2}\left(\Gamma_{0}\right)$ to $l^{2}\left(\Gamma_{1}\right)$; see [Popa 1994]. 
Conformal nets and subnets. We recall from [Guido and Longo 1996] the notion of an irreducible conformal net (or precosheaf) and representations thereof.

Let $\mathbb{G}_{0} \simeq \operatorname{PSL}(2, \mathbb{R})$ be the group of conformal (hence orientation-preserving) transformations of the Riemann sphere $\mathbb{C} \cup \infty$ that take the unit disk onto itself. Let $\mathbb{G}$ be the universal covering group of $\mathbb{G}_{0}$; this is a simple Lie group and acts on the unit circle $S^{1}$. By $R(\vartheta) \in \mathbb{G}$ we denote the rotation through an angle $\vartheta \in \mathbb{R}$.

By an interval we mean a nonempty open connected subset $I$ of $S^{1}$ whose complement has nonempty interior. We associate with $I$ a one-parameter subgroup $\left\{\Lambda_{I}(s): s \in \mathbb{R}\right\} \subset \mathbb{G}$ and an anticonformal (hence orientation-reversing) map $r_{I}$ of the Riemann sphere. The latter is uniquely determined by the property that it fixes each endpoint of $I$ and takes the midpoint of $I$ to the midpoint of the interval complementary to $I$. The subgroup $\left\{\Lambda_{I}(s): s \in \mathbb{R}\right\}$ is the component of the identity of the subgroup of $\mathbb{G}$ consisting of elements that fix each of the two endpoints of $I$; its parametrization is well-defined by the condition that $\Lambda_{I}(s)$ corresponds to the map $x \mapsto e^{s} x$ of the positive real axis $\mathbb{R}^{+}$under conjugation by a conformal map of the Riemann sphere taking $I$ onto $\mathbb{R}^{+}$and the unit disk to the upper half-plane.

The involution $\iota: \mathbb{G}_{0} \rightarrow \mathbb{G}_{0}$ given by $\iota(g)(z)=\overline{g(\bar{z})}$ for $g \in \mathbb{G}_{0}$ lifts to an involution of $\mathbb{G}$, also denoted by $\iota$. Thus (letting $\mathbb{Z}_{2}$ be generated by $\iota$ ) we can form the semidirect product $\mathbb{G} \times \mathbb{Z}_{2}$, a double cover of $\mathbb{G}$; by projection to $\mathbb{G}_{0} \times_{\imath} \mathbb{Z}_{2}$ (the group of all conformal and anticonformal maps preserving the unit disk globally), we classify elements of $\mathbb{G} \times_{\imath} \mathbb{Z}_{2}$ as orientation-preserving or orientation-reversing. A representation $U$ of this double cover by operators on a Hilbert space $\mathscr{H}$ is called unitary if $U(g)$ is unitary whenever $g$ is orientation-preserving. The representation is called antiunitary if $U(g)$ is antiunitary whenever $g$ is orientation-reversing.

Let $\mathscr{I}$ be the set of intervals of $S^{1}$. A conformal net $\mathscr{A}$ is a family of von Neumann algebras $\mathscr{A}(I)$, for $I \in \mathscr{I}$, on some fixed separable Hilbert space $\mathscr{H}$, together with a nontrivial unitary representation $U$ of $\mathbb{G}$ in $\mathscr{H}$, the whole setup satisfying the following axioms:

(A) Isotony. If $I_{1}, I_{2} \in \mathscr{I}$ and $I_{1} \subset I_{2}$, then $\mathscr{A}\left(I_{1}\right) \subset \mathscr{A}\left(I_{2}\right)$.

(B) Conformal invariance. $U(g) \mathscr{A}(I) U(g)^{*}=\mathscr{A}(g I)$ for $g \in \mathbb{G}, I \in \mathscr{I}$.

(C) Positivity of the energy. The one-parameter group $\{U(R(\vartheta)): \theta \in \mathbb{R}\}$ has a positive generator.

(D) Locality. If $I_{1}$ and $I_{2}$ are disjoint intervals, $\mathscr{A}\left(I_{1}\right)$ and $\mathscr{A}\left(I_{2}\right)$ commute.

(E) Existence of the vacuum. There exists a unit vector $\Omega$, called the vacuum vector, which is $U(\mathbb{G})$-invariant and cyclic for $\bigvee_{I \in \mathscr{I}} \mathscr{A}(I)$, the von Neumann algebra generated by all the $\mathscr{A}(I)$.

A conformal net $\mathscr{A}$ is irreducible if it satisfies the next condition as well:

(F) Irreducibility. The only $U(\mathbb{G})$-invariant vectors are the scalar multiples of $\Omega$. 
The name comes from the fact that if $\mathscr{A}$ is irreducible, $\bigvee_{I \in \mathscr{I}} A(I)$ equals $B(\mathscr{H})$, the algebra of bounded operators on $\mathscr{H}$. See [Guido and Longo 1996, Proposition 1.2].

Proposition 2.1 [Guido and Longo 1996, Proposition 1.1]. Let $A$ be an irreducible conformal net with unitary representation $U$ and vacuum vector $\Omega$.

(a) Reeh-Schlieder theorem: $\Omega$ is cyclic and separating for each von Neumann algebra $A(I), I \in \mathscr{I}$.

(b) Bisognano-Wichmann property: $U$ extends to an antiunitary representation of $\mathbb{G} \times_{l} \mathbb{Z}_{2}$ such that $U\left(\Lambda_{I}(2 \pi t)\right)=\Delta_{I}^{i t}$ and $U\left(r_{I}\right)=J_{I}$ for any $I \in \mathscr{I}$, where $\Delta_{I}$ and $J_{I}$ are the modular operator and the modular conjugation associated with the algebra $\mathscr{A}(I)$ and the vector $\Omega$. Moreover, $U(g) \mathscr{A}(I) U(g)^{*}=\mathscr{A}(g I)$ for any $g \in \mathbb{G} \times{ }_{1} \mathbb{Z}_{2}$.

(c) Additivity: if a family of intervals $I_{i}$ covers $I$, then $\mathscr{A}(I) \subset \bigvee_{i} \mathscr{A}\left(I_{i}\right)$.

(d) Haag duality: $\mathscr{A}(I)^{\prime}=\mathscr{A}\left(I^{\prime}\right)$, where $I^{\prime}$ is the interior of $S^{1} \backslash I$ and the 'on the left means the commutant as usual.

An irreducible conformal net $\mathscr{A}$ is strongly additive if $\mathscr{A}\left(I_{1}\right) \vee \mathscr{A}\left(I_{2}\right)=\mathscr{A}(I)$ whenever $I, I_{1}, I_{2} \in \mathscr{I}$ with $I=I_{1} \cup\{x\} \cup I_{2}$ for some $x$. The net $\mathscr{A}$ is split if, whenever $I_{1}, I_{2} \in \mathscr{I}$ are closure-disjoint (meaning that $\bar{I}_{1} \cap \bar{I}_{2}=\varnothing$ ), the von Neumann algebras $\mathscr{A}\left(I_{1}\right) \vee \mathscr{A}\left(I_{2}\right)$ and $\mathscr{A}\left(I_{1}\right) \otimes \mathscr{A}\left(I_{2}\right)$ are naturally isomorphic. Denote by $\mathscr{I}_{2}$ the set of unions of two closure-disjoint elements of $\mathscr{I}$, as in [Kawahigashi et al. 2001]. If $E=I_{1} \cup I_{2} \in \mathscr{I}_{2}$ and if $I_{3} \cup I_{4} \in \mathscr{I}_{2}$ is the interior of $S^{1} \backslash E$, put $\mathscr{A}(E):=A\left(I_{1}\right) \vee A\left(I_{2}\right)$ and $\hat{A}(E):=\left(A\left(I_{3}\right) \vee A\left(I_{4}\right)\right)^{\prime}$. Note that $\mathscr{A}(E) \subset \hat{A}(E)$.

Definition 2.2 (Complete rationality). $A$ is completely rational, or $\mu$-rational, if $\mathscr{A}$ is split, strongly additive, and the index $[\hat{\mathscr{A}}(E): \mathscr{A}(E)]$ is finite for some $E \in \mathscr{I}_{2}$. In this case $[\hat{\mathscr{A}}(E): \mathscr{A}(E)]$ does not depend on $E$ [Kawahigashi et al. 2001, Proposition 5]; it is called the $\mu$-index of $\mathscr{A}$ and denoted by $\mu_{\mathscr{A}}$.

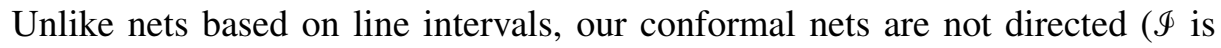
not directed under inclusion). Hence, when it comes to defining a representation of a conformal net $\mathscr{A}$, rather than representing a single algebra containing all the $\mathscr{A}(I)$, we need a whole family of representations, one for each $I$. More formally, if $\mathscr{A}$ is an irreducible conformal net with associated data $\mathcal{H}, U, \Omega$, a representation $\pi$ of $\mathscr{A}$ is a family $\left(\pi_{I}\right)_{I \in \mathscr{I}}$, where each $\pi_{I}$ is a representation of $\mathscr{A}(I)$ on a fixed separable Hilbert space $\mathscr{H}_{\pi}$, subject to an isotony condition

$$
\left.\pi_{I_{2}}\right|_{\mathscr{A}\left(I_{1}\right)}=\pi_{I_{1}} \quad \text { for } I_{1} \subset I_{2} .
$$

A covariant representation $\pi$ of $\mathscr{A}$ is a representation $\pi$ of $\mathscr{A}$, together with a unitary representation $U_{\pi}$ of $\mathbb{G}$ of positive energy (see (C) above), such that

$$
\operatorname{Ad} U_{\pi}(g) \cdot \pi_{I}=\pi_{g I} \cdot \operatorname{Ad} U(g) .
$$


A covariant representation $\pi$ is irreducible if $\bigvee_{I \in \mathscr{I}} \pi(\mathscr{A}(I))=B\left(\mathscr{H}_{\pi}\right)$. Clearly an irreducible conformal net $\mathscr{A}$ can be regarded as an irreducible representation of itself; we call this the vacuum representation.

By [Guido and Longo 1992], a representation that has finite index is covariant.

Let $H$ be a connected, simply laced compact Lie group. By [Gabbiani and Fröhlich 1993, Theorem 3.2], the vacuum positive-energy representation of the loop group $L H$ [Pressley and Segal 1986] at level $k$ gives rise to an irreducible conformal net, denoted by $\mathscr{A}_{H_{k}}$. By [Gabbiani and Fröhlich 1993, Theorem 3.3], every irreducible positive-energy representation of the loop group $L H$ at level $k$ gives rise to an irreducible covariant representation of $\mathscr{A}_{H_{k}}$. The vacuum representation of the Virasoro algebra with central charge $c_{0}>0$ also gives rise to a conformal net, denoted by $\mathscr{A}_{c=c_{0}}$ [Gabbiani and Fröhlich 1993, §3]. We will see such examples in Section 4.

Given a fixed $\xi \in S^{1}$, the set of intervals $I$ with $\bar{I} \subset S^{1} \backslash \xi$ is denoted by $\rrbracket_{\xi}$; this is a directed set under inclusion. Let $U_{\mathscr{A}}$ be the associated quasilocal $C^{*}$ algebra $\varkappa_{\mathscr{A}}=\overline{\bigcup_{I \in \mathscr{I}_{\xi}} \mathscr{A}(I)}$ (norm closure). Any representation $\lambda$ of $\mathscr{A}$ localized on $I \in \mathscr{I}_{\xi}$ (that is, one whose restriction to $\mathscr{A}\left(I^{\prime}\right)$ is the identity) restricts to a DHR endomorphism of $U_{\mathscr{A}}$ localized on $I$, also denoted by $\lambda$, and vice versa [Longo 2003, Proposition 11]. We will use these two descriptions interchangeably without further notice.

Fix $I \in I_{\xi}$. Let $\lambda, \mu$ be representations of $\mathscr{A}$. Choose $I_{-}, I_{+} \in I_{\xi}$ disjoint from $I$, so that $I_{-}$lies counterclockwise from $I$ and $I_{+}$lies clockwise from $I$. Choose covariant representations $\hat{\lambda}_{+}, \hat{\lambda}_{-}$of $\mathscr{A}$ unitarily equivalent to $\lambda$ but localized on $I_{+}, I_{-}$respectively and let $u_{+}, u_{-}$be unitary intertwiners from $\lambda_{+}, \lambda_{-}$to $\mu$. (We will not distinguish between local and global intertwiners since they are the same when $\mathscr{A}$ is strongly additive.) The braiding operators are defined by

$$
\epsilon(\lambda, \mu):=\mu\left(u_{+}^{*}\right) u_{+}, \quad \tilde{\epsilon}(\lambda, \mu)=\mu\left(u_{-}^{*}\right) u_{-} .
$$

These are elements of $\mathscr{A}(I)$ and do not depend on the choice of $u_{+}, u_{-}, I_{+}, I_{-}$. They satisfy the Yang-Baxter equation (YBE) and the braiding-fusion equation (BFE). See [Xu 1998; Böckenhauer and Evans 1998] for this and other properties of the braiding operators.

By a conformal subnet of $\mathscr{A}$ [Longo 2003] we mean a map

$$
I \in \mathscr{I} \rightarrow \mathscr{B}(I) \subset \mathscr{A}(I)
$$

that associates to each $I \in \mathscr{I}$ a von Neumann subalgebra $\mathscr{B}(I)$ so that isotony and covariance with respect to $U$ hold (conditions (A) and (B) of page 170 , with $\mathscr{B}$ instead of $\mathscr{A}$ ). When restricted to $\mathscr{I}_{\xi}$, the conformal nets $\mathscr{B} \subset \mathscr{A}$ form a standard net of inclusions as defined in [Longo and Rehren 1995, §3.1]. 
Proposition 2.3 [Kawahigashi et al. 2001; Xu 2001, Proposition 2.4]. Suppose $\mathscr{B} \subset \mathcal{A}$ is a standard net of inclusions as defined in [Longo and Rehren 1995, 3.1]. Let $E \in \mathscr{I}_{2}$. If $\mathscr{A} \subset \mathscr{B}$ has finite index $[\mathscr{A}: \mathscr{B}]$ and $\mathscr{A}$ and $\mathscr{B}$ are split, then

$$
[\hat{\mathscr{B}}(E): \mathscr{B}(E)]=[\mathscr{A}: \mathscr{B}]^{2}[\hat{\mathscr{A}}(E): \mathscr{A}(E)] .
$$

Orbifolds. Let $\mathscr{A}$ be an irreducible conformal net on a Hilbert space $\mathscr{H}$ and let $G$ be a compact group. Let $V: G \rightarrow U(\mathscr{H})$ be a faithful unitary representation of $G$ on $\mathscr{H}$. (If $V: G \rightarrow U(\mathscr{H})$ is not faithful, we can replace $G$ by $G / \operatorname{ker} V$.)

Definition 2.4. We say that $G$ acts properly on $\mathscr{A}$ if

(1) $\alpha_{g}(a):=V(g) a V\left(g^{*}\right) \in \mathscr{A}(I)$ for every $I \in \mathscr{I}, a \in \mathscr{A}(I)$ and $g \in G$, and

(2) $V(g) \Omega=\Omega$ for every $g \in G$.

Suppose a finite group $G$ acts properly on $\mathscr{A}$. For each interval $I$, define

$$
\mathscr{B}(I):=\left\{a \in \mathscr{A}(I) \mid V(g) a V\left(g^{*}\right)=a \text { for all } g \in G\right\} .
$$

Let $\mathscr{H}_{0}=\{x \in \mathscr{H} \mid V(g) x=x$ for all $g \in G\}$. The projection $P_{0}: \mathscr{H} \rightarrow \mathscr{H}_{0}$ commutes with every element of $\mathscr{B}(I)$ and with $U(g)$, for all $g \in \mathbb{G}$.

Define $\mathscr{A}^{G}(I):=\mathscr{B}(I) P_{0}$ on $\mathscr{H}_{0}$. The unitary representation $U$ of $\mathbb{G}$ on $\mathscr{H}$ restricts to a unitary representation (still denoted by $U$ ) of $\mathbb{G}$ on $\mathscr{H}_{0}$. By $[\mathrm{Xu}$ 2001, Proposition 2.2] the map $I \in \mathscr{I} \rightarrow \mathscr{A}^{G}(I)$ on $\mathscr{H}_{0}$ together with the unitary representation $U$ of $\mathbb{G}$ on $\mathscr{H}_{0}$ is an irreducible conformal net, denoted by $\mathscr{A}^{G}$. This is called the orbifold of $\mathscr{A}$ with respect to $G$.

The net $\mathscr{B} \subset \mathscr{A}$ is a standard net of inclusions when restricted to intervals in $\rrbracket_{\xi}$, with conditional expectation $E$ defined by

$$
E(a):=\int_{G} \alpha_{g}(a) d g \text { for all } a \in \mathscr{A}(I),
$$

where $d g$ is the normalized Haar measure on $G$.

Recall that $I^{\prime}$ denotes the interior of $S^{1} \backslash I$, where $I \in \mathscr{I}$.

Lemma 2.5. (1) For any interval $I, \mathscr{A}^{G}(I)^{\prime} \cap \mathscr{A}(I)=\mathbb{C}$.

(2) Let $I, I_{1}, I_{2} \in I$ with $I_{1} \cup I_{2}=I$ minus a point. Let $g_{n} \in \mathbb{G}$ be a sequence of elements such that $g_{n} I_{1}=I_{1}$ and $g_{n} I_{2}$ is an increasing sequence of intervals containing $I_{2}$ and exhausting $I_{1}^{\prime}$. Take $\left.x \in \mathscr{B}_{(1}\left(I_{1}\right)^{\prime} \cap \mathscr{A}_{(} I_{2}^{\prime}\right)$, and suppose y is a weak limit of a subsequence of $\operatorname{Ad}_{g_{k}}(x):=g_{k} x g_{k}^{*}$. Then $y=\langle x \Omega, \Omega\rangle \mathrm{id}$.

Proof. The first part is [Carpi 1999, Proposition 2.1] (see also [D'Antoni et al. 2001] for related results). To prove (2), note that $\operatorname{Ad}_{g_{k}}(x)$ is in $\mathscr{B}\left(I_{1}\right)^{\prime} \cap \mathscr{A}\left(g_{k} I_{2}\right)^{\prime}$, so if $y$ is a weak limit, $y$ lies in $\mathscr{B}\left(I_{1}\right)^{\prime} \cap \mathscr{A}\left(g_{k} I_{2}\right)^{\prime}$ for all $k$, and it follows that $y$ lies in $\mathscr{B}\left(I_{1}\right)^{\prime} \cap \mathscr{A}\left(I_{1}\right)^{\prime}=\mathbb{C}$ by (1). Since $g_{k} \Omega=\Omega$, we have $\langle y \Omega, \Omega\rangle=\langle x \Omega, \Omega\rangle$, so $y=\langle x \Omega, \Omega\rangle$ id. 
Theorem 2.6. Let $A$ be an irreducible conformal net and let $G$ be a compact group acting properly on $\mathscr{A}$. Suppose that $\mathscr{A}$ is strongly additive. Then $\mathscr{A}^{G}$ is also strongly additive.

Proof. Let $I, I_{1}, I_{2} \in \mathscr{I}$ with $I_{1} \cup I_{2}=I$ minus a point. To show that $\mathscr{A}^{G}$ is strongly additive, it is sufficient to show that $\mathscr{B}\left(I_{1}\right) \vee \mathscr{B}\left(I_{2}\right)=\mathscr{B}(I)$.

We now show that $\mathscr{B}\left(I_{1}\right) \vee \mathscr{A}\left(I_{2}\right)=\mathscr{A}(I)$, which is enough since applying $E-$ see $(2-1)$ - then yields the desired equality. Since $\mathscr{A}$ is strongly additive, our target equality is equivalent to $\mathscr{B}\left(I_{1}\right) \vee \mathscr{A}\left(I_{2}\right)=\mathscr{A}\left(I_{1}\right) \vee \mathscr{A}\left(I_{2}\right)$, or yet, by taking commutants and using Haag duality, to

$$
N:=\mathscr{B}\left(I_{1}\right)^{\prime} \cap \mathscr{A}\left(I_{2}^{\prime}\right)=\mathscr{A}\left(I_{1}^{\prime}\right) \cap \mathscr{A}\left(I_{2}^{\prime}\right)=: M .
$$

Define

$$
N_{0}:=\mathscr{B}\left(I_{1}\right)^{\prime} \supset \mathscr{A}\left(I_{1}^{\prime}\right)=: M_{0} .
$$

By [Izumi et al. 1998, Remark 4.5], $N_{0}$ can be identified with the cross product of $M_{0}$ with $G$. By [Haagerup 1978, Theorem 3.1](a), for each continuous, positive definite function $\phi$ on $G$ there is a unique $\sigma$-weakly continuous linear map $E_{\phi}$ on $N_{0}$ such that

$$
\begin{aligned}
E_{\phi}\left(m_{0} x m_{0}^{\prime}\right) & =m_{0} E_{\phi}(x) m_{0}^{\prime} & & \text { for all } m_{0}, m_{0}^{\prime} \in M_{0}, \\
E_{\phi}(g) & =\phi(g) g & & \text { for all } g \in G .
\end{aligned}
$$

Let $g_{n} \in \mathbb{G}$ be a sequence as in part (2) of Lemma 2.5. Consider (for some $k \in \mathbb{N}$ )

$$
F_{\phi}\left(n_{0}\right):=\operatorname{Ad}_{g_{k}^{*}} E_{\phi}\left(\operatorname{Ad}_{g_{k}} n_{0}\right) \text { for all } n_{0} \in N_{0} .
$$

Note that

$$
\begin{aligned}
F_{\phi}\left(m_{0} x m_{0}^{\prime}\right) & =m_{0} F_{\phi}(x) m_{0}^{\prime}, & & \text { for all } m_{0}, m_{0}^{\prime} \in M_{0}, \\
F_{\phi}(g) & =\phi(g) g & & \text { for all } g \in G .
\end{aligned}
$$

Since $M_{0} g M_{0}$ is weakly closed in $N_{0}$, it follows that $E_{\phi}=F_{\phi}$, i.e.,

$$
E_{\phi}\left(\operatorname{Ad}_{g_{k}} n_{0}\right)=\operatorname{Ad}_{g_{k}}\left(E_{\phi}\left(n_{0}\right)\right) \quad \text { for all } n_{0} \in N_{0} .
$$

Let $x \in N$. Since $\mathscr{A}\left(I_{2}\right) \subset \mathscr{A}\left(I_{1}^{\prime}\right)$ we have $E_{\phi}=\phi(1)$ id on $\mathscr{A}\left(I_{1}^{\prime}\right)$, hence $E_{\phi}(x) \in N$. Let $a, b \in M$. Then

$$
\begin{aligned}
\left\langle\phi(1)^{-1} E_{\phi}(x) a \Omega, b \Omega\right\rangle & =\left\langle\phi(1)^{-1} E_{\phi}\left(b^{*} x a\right) \Omega, \Omega\right\rangle \\
& =\left\langle\phi(1)^{-1} \operatorname{Ad}_{g_{k}} E_{\phi}\left(b^{*} x a\right) \Omega, \Omega\right\rangle \\
& =\left\langle\phi(1)^{-1} E_{\phi}\left(\operatorname{Ad}_{g_{k}}\left(b^{*} x a\right)\right) \Omega, \Omega\right\rangle .
\end{aligned}
$$

By Lemma 2.5, there is a subsequence of $\operatorname{Ad}_{g_{k}} b^{*} x a$ that converges weakly to $\left\langle b^{*} x a \Omega, \Omega\right\rangle$. Since $E_{\phi}$ is weakly continuous, we must have

$$
\left\langle\phi(1)^{-1} E_{\phi}(x) a \Omega, b \Omega\right\rangle=\langle x a \Omega, b \Omega\rangle .
$$


Since $M \Omega$ is dense in $H$, we have $E_{\phi}(x)=\phi(1) x$. Choose $x$ to be a projection in $N$. By [Haagerup 1978, Theorem 3.1(b)] we have

$$
T(x)=\sup _{\phi \ll \delta} E_{\phi}(x)
$$

where $T$ is the operator valued weight from $N_{0}$ to $M_{0}$, and we write $\phi \ll \delta$ if $\phi$ is less than the Dirac measure in the unit element of $G$ with respect to the ordering of positive definite measures on $G$. So when $G$ is a finite group, $T(x)=x$, implying that $x \in M_{0} \cap N=M$. When $G$ is an infinite group, $T(x)=x \cdot \infty$, in the notation of [Strătilă 1981, p. 150]. Thus $x \in M_{0} \cap N=M$. So we have shown that any projection in $N$ is a projection in $M$, and so $M=N$.

Remark 2.7. The proof given is different from the one in [Xu 2001]. The same idea also gives a different proof of the result in [Longo 2003, §3.5.2] under the assumption that $\mathscr{B} \subset \mathscr{A}$ has finite index but without the assumption that $\mathscr{A}$ is split, if we modify the proof as follows. Instead of using $E_{\phi}$ we use $E$, the minimal conditional expectation from $N_{0}$ to $M_{0}$, which exists by the finite-index assumption. We check that, just as for $E_{\phi}$, we have

$$
E\left(\operatorname{Ad}_{g_{k}} n_{0}\right)=\operatorname{Ad}_{g_{k}} E\left(n_{0}\right) \quad \text { for all } n_{0} \in N_{0} .
$$

Note that $\operatorname{Ad}_{g_{k}^{*}} E_{\phi}\left(\operatorname{Ad}_{g_{k}} \cdot\right)$ is a conditional expectation from $N_{0}$ to $M_{0}$, and since $M_{0} \subset N_{0}$ is irreducible [Longo 2003, Lemma 14], we must have $\operatorname{Ad}_{g_{k}^{*}} E\left(\operatorname{Ad}_{g_{k}} \cdot\right)=$ $E(\cdot)$. Now using $E$ instead of $E_{\phi}$, the rest of the proof goes through, and we get $E(x)=x$ for all $x \in N$, so $x \in N \cap M_{0}=M$, i.e., $N=M$.

We now consider a large class of examples where Theorem 2.6 can be applied. Let $\mathscr{A}_{H_{k}}$ be the conformal net associated with representations of the loop group $L H$ at level $k$ (page 172). Let $G \subset H$ be any closed subgroup. By [Laredo 1997] $\mathscr{A}_{H_{k}}$ is strongly additive, and it is easy to check that $G$ (or its quotient by the kernel of the representation, see observation just before Definition 2.4) acts properly on $\mathscr{A}_{H_{k}}$. It follows that the fixed point net $\mathscr{A}_{H_{k}}^{G^{\prime}}$ is strongly additive by Theorem 2.6. We will see a special case of such examples in Section 4.

\section{Induction and strongly additive pairs}

Induction of a pair. Let $\mathscr{B} \subset \mathscr{A}$ be a pair of conformal nets, with $\mathscr{B}$ strongly additive. Fix a point $\xi \in S^{1}$. All the intervals in this section will be in $\Phi_{\xi}$ unless otherwise stated.

For $I \subset J \in \mathscr{I}_{\xi}$, we denote by $\gamma_{\mathscr{A}}$ the canonical endomorphism $\mathscr{A}(J) \rightarrow \mathscr{B}(J)$, which extends the canonical endomorphism $\mathscr{A}(I) \rightarrow \mathscr{B}(I)$ [Longo and Rehren 1995, Corollary 3.3]. The restriction of $\gamma_{\mathscr{A}}$ to $\mathscr{B}$ will be denoted simply by $\gamma$ (when no confusion arises, we may also denote $\gamma_{\mathscr{A}}$ by $\gamma$ ). Note that $\gamma$ is a (DHR) 
representation of $\mathscr{B}$ which is unitarily equivalent to the defining representation of $\mathscr{B}$ on the vacuum Hilbert space of $\mathscr{A}$, since $\mathscr{B}$ is assumed to be strongly additive; see the proof of [Longo 2003, Proposition 17].

The following is essentially [Longo and Rehren 1995, Proposition 3.9], except that under our conditions we have further properties.

Proposition 3.1. Let $\mathscr{B} \subset A$ be a pair of conformal nets with $\mathscr{B}$ strongly additive. Fix $I \subset g_{\xi}$. With every representation $\lambda$ of $\mathscr{B}$ associate

$$
\alpha_{\lambda}:=\gamma_{\mathscr{A}}^{-1} \operatorname{Ad}_{\epsilon} \lambda \gamma_{\mathscr{A}}, \quad \tilde{\alpha}_{\lambda}:=\gamma_{\mathscr{A}}^{-1} \operatorname{Ad}_{\tilde{\epsilon}} \lambda \gamma_{\mathscr{A}},
$$

where $\epsilon=\epsilon(\lambda, \gamma)$ and $\tilde{\epsilon}:=\tilde{\epsilon}(\lambda, \gamma)$ are the braiding operators in $\mathscr{B}(I)$. Then $\alpha_{\lambda}(\mathscr{A}(I)) \subset \mathscr{A}(I)$ and $\alpha_{\lambda}=\lambda$ on $\mathscr{B}(I)$. Moreover, $\alpha_{\lambda}$ is localized on $I$ if and only if

$$
\epsilon(\lambda, \gamma) \epsilon(\gamma, \lambda)=\mathrm{id}
$$

Proof. Choose a unitary intertwiner $u$ transporting $\lambda$ to $\hat{\lambda}$ localized on $I_{+}$, so that $\epsilon(\lambda, \gamma)=\gamma\left(u^{*}\right) u$. Then, for any $x \in \mathscr{A}(I)$,

$$
\operatorname{Ad}_{\epsilon} \lambda \gamma(x)=\operatorname{Ad}_{\gamma\left(u^{*}\right)} \hat{\lambda} \gamma=\gamma\left(\operatorname{Ad}_{u^{*}} x\right) .
$$

Let $J$ be an interval in $\Im_{\xi}$ containing $I \cup I_{+}$. Then $\gamma\left(\operatorname{Ad}_{u^{*}} x\right) \in \gamma(\mathscr{A}(J))$. The lefthand side of this relation depends on $J$ only through the braiding operator $\epsilon$, and by the invariance property of $\epsilon$ we can choose a decreasing sequence of intervals $J_{n} \supset \bar{I}$ such that $\bigcap_{n} J_{n}=I$. By Proposition 2.1(c) we have $\bigcap_{n} \mathscr{A}\left(J_{n}\right)=\mathscr{A}(I)$. It follows that $\bigcap_{n} \gamma\left(\mathscr{A}\left(J_{n}\right)\right)=\gamma(\mathscr{A}(I))$, so we have shown that

$$
\operatorname{Ad}_{\epsilon} \lambda \gamma(\mathscr{A}(I)) \subset \gamma(\mathscr{A}(I)) .
$$

Hence $\alpha_{\lambda}(\mathscr{A}(I)) \subset \mathscr{A}(I)$ and

$$
\alpha_{\lambda}(x)=u^{*} x u \quad \text { for all } x \in \mathscr{A}(I)
$$

A similar formula holds for $\tilde{\alpha}_{\lambda}$ if we choose the unitary intertwiner accordingly. The rest of the proof follows that of [Longo and Rehren 1995, Proposition 3.9].

The notation $\alpha_{\lambda}$ of (3-1) was introduced in [Böckenhauer and Evans 1998]. We refine it to $\alpha_{\lambda}^{\Re \rightarrow A}$ when there may be ambiguity as to which pair is meant.

In [Xu 1998], a slightly different induction $a_{\lambda} \in$ End $\mathscr{B}(I)$ was used, motivated by certain questions in subfactors; the relations between $a_{\lambda}$ and $\alpha_{\lambda}$ are given in [Xu 2005]. We point out one basic relation. Let $\rho \in \operatorname{End} \mathscr{B}(I)$ be such that

$$
\rho(\mathscr{B}(I))=\gamma(\mathscr{A}(I)) \text { and } \rho \bar{\rho}=\gamma .
$$

Then

$$
a_{\lambda}(\gamma(a))=\rho^{-1}\left(\gamma \alpha_{\lambda}(a)\right) \quad \text { for all } a \in \mathscr{A}(I) .
$$

Using this relation one can rewrite all the results of this section in terms of $a_{\lambda}$. 
The next lemma is implicitly contained in [Longo and Rehren 1995].

Lemma 3.2. (1) If $x \in \operatorname{Hom}(\lambda, \mu)_{\mathscr{B}}$, then $x \in \operatorname{Hom}\left(\alpha_{\lambda}, \alpha_{\mu}\right)_{\mathscr{A}}$.

(2) $\alpha_{\lambda \mu}=\alpha_{\lambda} \alpha_{\mu}$.

(3) If $[\delta]=[\lambda]+[\mu]$, then $\left[\alpha_{\delta}\right]=\left[\alpha_{\lambda}\right]+\left[\alpha_{\mu}\right]$.

Proof. Let $u_{\lambda}$ and $u_{\mu}$ be unitary intertwiners from $\lambda$ and $\mu$ localized on $I$ to $\hat{\lambda}$ and $\hat{\mu}$ localized on $I_{+}$. We can choose $I_{+}$to share only one boundary point with

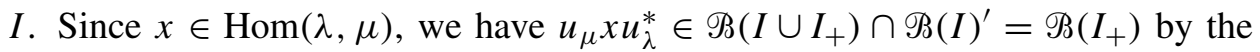
strong additivity of $\mathscr{B}$. It follows that $u_{\mu} x u_{\lambda}^{*}$ commutes with every element of $\mathscr{A}(I)$, and (1) is proved. (2) and (3) follow from the definitions, YBE and BFE as in [Böckenhauer and Evans 1998, §3].

Lemma 3.3. Let $\mathscr{\Re} \subset A$ be a pair of conformal nets with $\mathscr{B}$ strongly additive.

(1) If $\lambda$ is a representation of $\mathscr{B}$ localized on I and $\sigma \in$ End $\mathscr{A}(I)$, then

$$
\left\langle\alpha_{\lambda}, \sigma\right\rangle_{\mathscr{A}} \leq\langle\lambda, \gamma \sigma\rangle_{\mathscr{B}} .
$$

(2) Suppose $\alpha_{\lambda}, \alpha_{\mu}$ are localized on I, and denote by $\epsilon\left(\alpha_{\lambda}, \alpha_{\mu}\right)$ the braiding operator of $\alpha_{\lambda}, \alpha_{\mu}$ considered as (DHR) representations of $\mathcal{A}$. Then

$$
\epsilon\left(\alpha_{\lambda}, \alpha_{\mu}\right)=\epsilon(\lambda, \mu) .
$$

(3) For an intermediate net $\mathscr{b}$ such that the pairs $\mathscr{B} \subset \mathscr{C}$ and $\mathscr{C} \subset \mathscr{A}$ are conformal, suppose $\lambda_{1}:=\alpha_{\lambda}^{\Re \rightarrow \mathscr{C}}$ is localized on I. Then

$$
\alpha_{\lambda_{1}}^{\mathscr{C} \rightarrow \mathscr{A}}(a)=\alpha_{\lambda}^{\mathscr{\Re} \rightarrow A}(a) \quad \text { for all } a \in \mathscr{A}(I) .
$$

Proof. (1) Let $E: \mathscr{A}(I) \rightarrow \mathscr{B}(I)$ be the faithful conditional expectation. Let $v \in$ $\operatorname{Hom}(\mathrm{id}, \gamma)_{\mathscr{B}}$ be the isometry such that $E(\cdot)=v^{*} \gamma(\cdot) v$; see [Longo and Rehren 1995]. For any $x \in \operatorname{Hom}\left(\alpha_{\lambda}, \sigma\right)_{\mathscr{A}}$, it is easy to check that $\gamma(x) v \in \operatorname{Hom}(\lambda, \gamma \sigma)_{\mathscr{B}}$. To prove (1), we just have to show that the linear map

$$
x \in \operatorname{Hom}\left(\alpha_{\lambda}, \sigma\right)_{\mathscr{A}} \rightarrow \gamma(x) v \in \operatorname{Hom}(\lambda, \gamma \sigma)_{\mathscr{B}}
$$

is one-to-one. Assume that $\gamma(x) v=0$. Then $E\left(x^{*} x\right)=v^{*} \gamma\left(x^{*}\right) v \gamma(x) v=0$. It follows that $x=0$ since $E$ is faithful.

(2), (3) Let $u_{\lambda}$ be a unitary intertwiner from $\lambda$ to $\hat{\lambda}$. By Lemma 3.2(1), $u_{\lambda}$ is also a unitary intertwiner from $\alpha_{\lambda}$ to $\alpha_{\hat{\lambda}}$. So

$$
\epsilon\left(\alpha_{\lambda}, \alpha_{\mu}\right)=\alpha_{\mu}\left(u_{\lambda}\right)^{*} u_{\lambda}=\mu\left(u_{\lambda}\right)^{*} u_{\lambda}=\epsilon(\lambda, \mu)
$$

and, by (3-2) in the proof of Proposition 3.1,

$$
\alpha_{\lambda_{1}}^{\mathscr{C} \rightarrow \mathscr{A}}(a)=u_{\lambda}^{*}(a) u_{\lambda}=\alpha_{\lambda}^{\mathscr{P} \rightarrow \mathscr{A}}(a) \quad \text { for all } a \in \mathscr{A}(I)
$$


Note that by Proposition 3.1, $\alpha_{\lambda} \in \operatorname{End} \mathscr{A}(I)$.

Proposition 3.4. Let $\mathscr{A}, \mathscr{B}$ and $\lambda$ be as before, and suppose $\lambda$ has finite index.

(1) $d_{\lambda}$ equals $d_{\alpha_{\lambda}}$, the statistical dimension of $\alpha_{\lambda}$.

(2) (Commuting squares) If $E$ is the conditional expectation from $\mathscr{A}(I)$ to $\mathscr{B}(I)$ and $F_{\lambda}$ the minimal conditional expectation from $\mathscr{A}(I) \rightarrow \alpha_{\lambda}(\mathscr{A}(I))$, then $E F_{\lambda}=F_{\lambda} E$.

Proof. First part of (1): $d_{\alpha_{\lambda}} \leq d_{\lambda}$. We give two proofs of this inequality.

The first uses additivity in a similar way as in the proof of Proposition 3.1. Let $\epsilon:=\epsilon(\lambda, \gamma)=\gamma\left(u^{*}\right) u$ and $\bar{\epsilon}:=\epsilon(\bar{\lambda}, \gamma)=\gamma\left(\bar{u}^{*}\right) \bar{u}$ be the braiding operators, where $u, \bar{u} \in \mathscr{B}(J)(J \supset I)$ are unitary intertwiners. Set $\lambda_{\epsilon}:=\operatorname{Ad}_{\epsilon} \lambda$ and $\bar{\lambda}_{\bar{\epsilon}}:=\operatorname{Ad}_{\bar{\epsilon}} \bar{\lambda}$, and let $E_{\lambda}: \mathscr{B}(I) \rightarrow \lambda_{\epsilon}(\mathscr{B}(I))$ be the unique minimal conditional expectation. We will prove that

$$
E_{\lambda}\left(\gamma(\mathscr{A}(I)) \subset \lambda_{\epsilon} \gamma(\mathscr{A}(I))\right.
$$

this suffices, because it implies that the minimal index of $\lambda_{\epsilon} \gamma(\mathscr{A}(I)) \subset \gamma(\mathscr{A}(I))$ (which equals $d_{\alpha_{\lambda}}^{2}$ since $\lambda_{\epsilon} \gamma=\gamma\left(\alpha_{\lambda}\right)$ ) is at most $d_{\lambda}^{2}$; that is, $d_{\alpha_{\lambda}}^{2} \leq d_{\lambda}^{2}$.

By [Longo 1992], there exists an isometry $v \in \operatorname{Hom}\left(\mathrm{id}, \bar{\lambda}_{\bar{\epsilon}} \lambda_{\epsilon}\right)$ such that

$$
E_{\lambda}(\cdot)=\lambda_{\epsilon}\left(v^{*}\right) \lambda_{\epsilon} \bar{\lambda}_{\bar{\epsilon}}(\cdot) \lambda_{\epsilon}(v) .
$$

Then $\lambda_{\epsilon}(b)=\gamma\left(u^{*}\right) b \gamma(u)$ and $\lambda_{\epsilon}(b)=\gamma\left(\bar{u}^{*}\right) b \gamma(\bar{u})$, so

$$
E_{\lambda}(\gamma(a))=\gamma\left(u^{*}\right) v^{*} \gamma\left(\bar{u}^{*} a \bar{u}\right) v \gamma(u) .
$$

We show that $v \in \gamma(\mathscr{A}(I))$. Since $v \in \operatorname{Hom}\left(\mathrm{id}, \bar{\lambda}_{\bar{\epsilon}} \lambda_{\epsilon}\right)$, we have

$$
v b=\gamma\left(\bar{u}^{*} u^{*}\right) b \gamma(u \bar{u}) v \quad \text { for all } b \in \mathscr{B}(I)
$$

and so $\gamma(u \bar{u}) v \in \mathscr{B}(I)^{\prime} \cap \mathscr{B}(J)=\mathscr{B}\left(I_{+}\right)$, where the equality follows from the strong additivity of $\mathscr{B}$. Hence $v=\gamma\left(\bar{u}^{*} u^{*}\right) \gamma(u \bar{u}) v \in \gamma(\mathscr{B}(J))$, since $\gamma$ is localized on $I$. As in the proof of Proposition 3.1 we can choose a decreasing sequence $J_{n}$ such that $\bigcap_{n} J_{n}=I$ and we have $v \in \bigcap_{n} \gamma\left(\mathscr{B}\left(J_{n}\right)\right)=\gamma(\mathscr{B}(I))$. From the expressions for $E_{\lambda}(\gamma(a))$ above we have proved that

$$
E_{\lambda}(\gamma(\mathscr{A}(I))) \in \bigcap_{n} \gamma\left(\mathscr{A}\left(J_{n}\right)\right)=\gamma(\mathscr{A}(I)) .
$$

At the same time, if $\lambda_{\epsilon}(b)=\gamma(a)$, then $\gamma\left(u^{*}\right) b \gamma(u)=\gamma(a)$, and so $b=$ $\gamma\left(\right.$ uau $\left.^{*}\right) \in \gamma(\mathscr{A}(J))$; by the same argument as above, $b \in \gamma(\mathscr{A}(I))$. This shows that

$$
\gamma(\mathscr{A}(I)) \cap \lambda_{\epsilon}(\mathscr{B}(I))=\lambda_{\epsilon} \gamma(\mathscr{A}(I)) .
$$

Hence $E_{\lambda}\left(\gamma(\mathscr{A}(I)) \subset \gamma(\mathscr{A}(I)) \cap \lambda_{\epsilon}(\mathscr{B}(I))=\lambda_{\epsilon} \gamma(\mathscr{A}(I))\right.$, which is (3-3). 
Now we give a second proof of $d_{\alpha_{\lambda}} \leq d_{\lambda}$. Let $F_{\lambda}: \mathscr{B}(I) \rightarrow \lambda(\mathscr{B}(I))$ be the minimal conditional expectation. By [Longo 1992] (see also [Longo and Rehren 1995, 2.14]), $F_{\lambda}$ is induced by an isometry $R_{\bar{\lambda} \lambda} \in \operatorname{Hom}(\mathrm{id}, \bar{\lambda} \lambda)$ in the sense that

$$
F_{\lambda}(\cdot)=\lambda\left(R_{\bar{\lambda} \lambda}\right) \lambda \bar{\lambda}(\cdot) \lambda\left(R_{\bar{\lambda} \lambda}^{*}\right) .
$$

There is likewise an isometry $R_{\lambda \bar{\lambda}} \in \operatorname{Hom}(\mathrm{id}, \lambda \bar{\lambda})$, and these two isometries satisfy

$$
R_{\lambda \bar{\lambda}}^{*} \lambda\left(R_{\bar{\lambda} \lambda}\right)=R_{\bar{\lambda} \lambda}^{*} \bar{\lambda}\left(R_{\lambda \bar{\lambda}}\right)=\frac{1}{d(\lambda)} .
$$

Now Lemma 3.2(1) yields $R_{\bar{\lambda} \lambda} \in \operatorname{Hom}\left(\mathrm{id}, \alpha_{\bar{\lambda}} \alpha_{\lambda}\right)$ and $R_{\lambda \bar{\lambda}} \in \operatorname{Hom}\left(\mathrm{id}, \alpha_{\lambda} \alpha_{\bar{\lambda}}\right.$ ), and it follows from the properties of $R_{\bar{\lambda} \lambda}, R_{\lambda \bar{\lambda}}$ [Longo and Rehren 1995] that

$$
F_{\lambda}(\cdot)=\lambda\left(R_{\bar{\lambda} \lambda}\right) \alpha_{\lambda} \alpha_{\bar{\lambda}}(\cdot) \lambda\left(R_{\bar{\lambda} \lambda}^{*}\right)
$$

is a conditional expectation from $\mathscr{A}(I) \rightarrow \alpha_{\lambda}(\mathscr{A}(I))$ with index $d_{\lambda}^{2}$. Hence $d_{\alpha_{\lambda}} \leq d_{\lambda}$. Second part of (1): $d_{\alpha_{\lambda}} \geq d_{\lambda}$.

Let $\Gamma$ be the principal graph of $\lambda$ and $\Gamma_{0}$ the set of even vertices. By Lemma 3.2 and the properties of the statistical dimension, $V:=\left(d_{\alpha_{x}}\right)_{x \in \Gamma_{0}}$ is a vector satisfying $\Gamma \Gamma^{t} V=d_{\alpha_{\lambda}} d_{\alpha_{\bar{\lambda}}} V$.

By [Popa 1994, Proposition 1.3.5],

$$
\|\Gamma\|^{2}=\lim _{n \rightarrow \infty}\left(\left(\Gamma \Gamma^{t}\right)^{n} \delta, \delta\right)^{1 / n},
$$

where $\delta \in l^{2}\left(\Gamma_{0}\right)$ is a vector which is 1 at the identity sector and 0 elsewhere. Hence

$$
\begin{aligned}
\|\Gamma\|^{2} & =\lim _{n \rightarrow \infty}\left(\left(\Gamma \Gamma^{t}\right)^{n} \delta, \delta\right)^{1 / n} \leq \lim _{n \rightarrow \infty}\left(\left(\Gamma \Gamma^{t}\right)^{n} \delta, V\right)^{1 / n} \\
& =\lim _{n \rightarrow \infty}\left(\delta,\left(\Gamma \Gamma^{t}\right)^{n} V\right)^{1 / n}=\lim _{n \rightarrow \infty}\left(\delta,\left(d_{\alpha_{\lambda}} d_{\alpha_{\bar{\lambda}}}\right)^{n} V\right)^{1 / n}=d_{\alpha_{\lambda}} d_{\alpha_{\bar{\lambda}}} .
\end{aligned}
$$

Since $\lambda$ is amenable (see [Longo and Roberts 1997, Theorem 5.31 and remarks on p. 122]), we have $d_{\lambda}^{2}=\|\Gamma\|^{2}$; thus $d_{\lambda}^{2} \leq d_{\alpha_{\lambda}} d_{\alpha_{\bar{\lambda}}}$ and so $d_{\lambda} \leq d_{\alpha_{\lambda}}$. Together with the reverse inequality, this concludes the proof of (1).

(2) Since $d_{\lambda}=d_{\alpha_{\lambda}}$, it follows that the $F_{\lambda}$ defined at the top of this page is the minimal conditional expectation from $\mathscr{A}(I) t_{0} \alpha_{\lambda}(\mathscr{A}(I))$.

For any $a \in \mathscr{A}(I)$, we have

$$
E\left(\alpha_{\lambda \bar{\lambda}}(a)\right)=E\left(u^{*} a u\right)=u^{*} E(a) u=\alpha_{\lambda \bar{\lambda}}(E(a)),
$$

where $u$ is the unitary intertwiner transporting $\lambda \bar{\lambda}$ to $\widehat{\lambda \bar{\lambda}}$, which is localized on $I_{+}$. Then

$$
\begin{aligned}
E\left(F_{\lambda}(a)\right) & =E\left(\lambda\left(R_{\bar{\lambda} \lambda}\right) \alpha_{\lambda} \alpha_{\bar{\lambda}}(a) \lambda\left(R_{\bar{\lambda} \lambda}^{*}\right)\right) \\
& \left.=\lambda\left(R_{\bar{\lambda} \lambda}\right) E\left(\alpha_{\lambda} \alpha_{\bar{\lambda}}(a)\right) \lambda\left(R_{\bar{\lambda} \lambda}^{*}\right)\right)=F_{\lambda}(E(a)) .
\end{aligned}
$$


Proposition 3.5. Suppose $\mu, \lambda$ are representations of $\mathscr{B}$ localized on $I$.

(1) Let $x$ be any subsector of $\alpha_{\mu}$. Then $\left[\alpha_{\lambda}\right][x]=[x]\left[\alpha_{\lambda}\right]$.

(2) Let $z$ and $y$ be subsectors of $\alpha_{\lambda}$ and $\tilde{\alpha}_{\mu}$ respectively. Then $[z][y]=[y][z]$.

Proof. (1) Let $\epsilon(\lambda, \mu)$ be the braiding operator. Then

$$
\alpha_{\mu} \alpha_{\lambda}=\alpha_{\operatorname{Ad}_{\epsilon(\lambda, \mu)} \lambda \mu}=\alpha_{\operatorname{Ad}_{\epsilon(\lambda, \mu)}} \alpha_{\lambda} \alpha_{\mu}=\operatorname{Ad}_{\epsilon(\lambda, \mu)} \alpha_{\lambda} \alpha_{\mu} .
$$

Now let $v_{x} \in \operatorname{Hom}\left(x, \alpha_{\mu}\right)$ be the isometry such that $x(\cdot)=v_{x}^{*} \alpha_{\mu}(\cdot) v_{x}$. As in the proof of [Xu 1998, Theorem 3.6, p. 377], it is sufficient to show that

$$
\alpha_{\lambda}\left(v_{x} v_{x}^{*}\right)=\epsilon(\lambda, \mu) v_{x} v_{x}^{*} \epsilon(\lambda, \mu)^{*}
$$

Applying $\gamma$ to the equality above, it is sufficient to show that

$$
\gamma \alpha_{\lambda}\left(v_{x} v_{x}^{*}\right)=\gamma\left(\epsilon(\lambda, \mu) v_{x} v_{x}^{*} \epsilon(\lambda, \mu)^{*}\right) .
$$

This follows from YBE and BFE as on [Xu 1998, p. 377].

(2) We first prove that $\operatorname{Ad}_{\epsilon(\lambda, \mu)} \alpha_{\lambda} \tilde{\alpha}_{\mu}=\tilde{\alpha}_{\mu} \alpha_{\lambda}$. Let $u_{\lambda+}$ and $u_{\mu-}$ be the intertwiners as in the definition of $\alpha_{\lambda}$ and $\tilde{\alpha}_{\mu}$ in Proposition 3.1. Then the desired equality is equivalent to

$$
\epsilon(\lambda, \mu) u_{\lambda+}^{*} u_{\mu-}^{*}=u_{\mu-}^{*} u_{\lambda+}^{*}
$$

Since $\epsilon(\lambda, \mu)=\mu\left(u_{\lambda+}^{*}\right) u_{\lambda+}$, we need to show that

$$
u_{\mu-} \mu\left(u_{\lambda+}^{*}\right) u_{\mu-}^{*}=u_{\lambda+}^{*},
$$

which follows from the fact that $u_{\lambda+}^{*} \in \mathscr{B}\left(I \cup I_{+}\right)$and $\hat{\mu}$ is localized on $I_{-}$.

The rest of the proof follows by YBE and BFE as in [Xu 1998, p. 385].

Many properties of relative braidings implicitly used in [Xu 1998] and further studied in [Böckenhauer and Evans 1999a] can also be proved in our current setting, but we will not use them in this paper.

Strongly additive pairs. Let $\mathscr{A}$ be a conformal net and let $\mathscr{B} \subset \mathscr{A}$ be a conformal subnet as defined in $\$ 2.1$. Motivated by the proof of Theorem 2.6, we define:

Definition. The pair $\mathscr{B} \subset \mathscr{A}$ is said to be strongly additive if

$$
\mathscr{B}\left(I_{1}\right) \vee \mathscr{A}\left(I_{2}\right)=\mathscr{A}(I)
$$

for any intervals $I, I_{1}, I_{2}$ such that $I_{1} \cup I_{2}=I$ minus one point.

This definition can be generalized to nets of algebras without conformal invariance, but conformal nets give most interesting examples of strongly additive pairs, so here we restrict our attention to them. In this case, by conformal invariance, it is sufficient to check the condition $\mathscr{B}\left(I_{1}\right) \vee \mathscr{A}\left(I_{2}\right)=\mathscr{A}(I)$ for a particular $I, I_{1}, I_{2}$. 
Lemma 3.6. (1) If the pair $\mathscr{B} \subset \mathscr{A}$ is strongly additive, then $\mathscr{B}$ and $\mathscr{A}$ are strongly additive, and

$$
\mathscr{B}\left(I_{1}\right)^{\prime} \cap \mathscr{A}\left(I_{1}\right)=\mathbb{C} \text { for all } I_{1} .
$$

(2) If $G$ is a compact group acting properly on $\mathscr{A}$ and $\mathscr{B}$ is the fixed-point subnet under the action of $G$, the pair $\mathscr{\Re} \subset A$ is strongly additive if and only if either $A$ is strongly additive or $\Re$ is strongly additive.

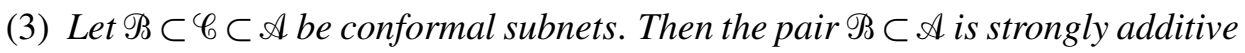
if and only if the pairs $\mathscr{B} \subset \mathscr{C}$ and $\mathscr{C} \subset A$ are strongly additive.

Proof. (1) The first statement follows trivially from the definition and the application of conditional expectation from $\mathscr{A}$ to $\mathscr{B}$. For the second, choose $I_{2}$ sharing only one boundary point with $I_{1}$, and let $I$ be the smallest interval containing $I_{1} \cup I_{2}$. By the assumption, $\mathscr{B}\left(I_{1}\right) \vee \mathscr{A}\left(I^{\prime}\right)=\mathscr{A}\left(I_{2}^{\prime}\right)$. Taking the commutants and applying Haag duality, we get $\mathscr{B}\left(I_{1}\right)^{\prime} \cap \mathscr{A}(I)=\mathscr{A}\left(I_{2}\right)$, so

$$
\mathscr{B}\left(I_{1}\right)^{\prime} \cap \mathscr{A}\left(I_{1}\right) \subset \mathscr{A}\left(I_{2}\right)
$$

for all $I_{2}$ sharing only one boundary point with $I_{1}$. Choosing a sequence of $I_{2}^{(n)}$ so that $\bigcap_{n} I_{2}^{(n)}$ is one point, by Proposition 2.1(c) we get

$$
\mathscr{B}\left(I_{1}\right)^{\prime} \cap \mathscr{A}\left(I_{1}\right) \subset \bigcap_{n} \mathscr{A}\left(I_{2}^{(n)}\right)=\mathbb{C} .
$$

(2) By Theorem 2.6 and part (1), it is sufficient to show that if $\mathscr{B}$ is strongly additive, so is the pair $\mathscr{B} \subset \mathscr{A}$. Let $I$ be an arbitrary interval, and $I_{1}, I_{2}$ are the connected components of a set obtained from $I$ by removing an interior point of $I$. Then

$$
\mathscr{B}(I) \subset \mathscr{B}\left(I_{1}\right) \vee \mathscr{A}\left(I_{2}\right) \subset \mathscr{A}(I),
$$

where the first inclusion follows from the strong additivity of $\mathscr{B}$. By [Izumi et al. 1998], there exists a closed subgroup $G_{1}$ of $G$ such that $\mathscr{B}\left(I_{1}\right) \vee \mathscr{A}\left(I_{2}\right)$ is the fixedpoint subalgebra of $\mathscr{A}(I)$ under the action of $G_{1}$. It follows that there is normal faithful conditional expectation form $\mathscr{A}(I)$ to $\mathscr{B}\left(I_{1}\right) \vee \mathscr{A}\left(I_{2}\right)$ preserving the vector state $(\cdot \Omega, \Omega)$. Since $\mathscr{A}\left(I_{2}\right) \Omega$ is dense in $H$ by the Reeh-Schlieder theorem in Proposition 2.1(a), it follows that

$$
\mathscr{B}\left(I_{1}\right) \vee \mathscr{A}\left(I_{2}\right)=\mathscr{A}(I)
$$

by Takesaki's theorem; see [Strătilă 1981, §9].

(3) This follows directly from the definitions and applying suitable conditional expectations.

As discussed in Section 2, orbifolds are examples of strongly additive pairs. More will be given later (page 187) as a result of the following proposition: 
Proposition 3.7. Let $\mathscr{A}$ be a conformal net and $\mathscr{P} \subset \mathcal{A}$ a conformal subnet. Assume that $\mathscr{B}$ is strongly additive and $U(g) \in \bigvee_{I} \mathscr{B}(I)$ for all $g \in \mathbb{G}$ where $U$ is the representation of the conformal group $\mathbb{G}$. Then the pair $\mathscr{B} \subset \mathcal{A}$ is strongly additive.

Proof. Let $I, I_{1}, I_{2}$ be intervals with $I_{1} \cup I_{2}=I$ minus one point. Let $E$ be the unique conditional expectation from $\mathscr{A}(I)$ to $\mathscr{B}(I)$ such that $\psi(E(\cdot))=\psi(\cdot)$, where $\psi(\cdot)=(\cdot \Omega, \Omega)$ is the normal faithful state on $\mathscr{A}(I)$ and $\Omega$ is the vacuum vector. Denote by $\Delta_{\psi}$ and $\Delta$ the modular operator of $\mathscr{A}(I)$ and $\mathscr{B}(I) \vee \mathscr{A}\left(I_{1}\right)$ with respect to $\Omega$. Notice that $\operatorname{Ad}_{\Delta_{\psi}^{i t}}$ and $\operatorname{Ad}_{\Delta^{i t}}$, for $t \in \mathbb{R}$, induce the same automorphism on $\mathscr{B}(I)$ and $\mathscr{B}\left(I^{\prime}\right)$, so

$$
\Delta_{\psi}^{i t} \Delta^{-i t} \in \mathscr{B}(I)^{\prime} \cap \mathscr{B}\left(I^{\prime}\right)^{\prime} .
$$

The geometric nature of $\Delta_{\psi}^{i t}$ (Proposition 2.1) and our assumption imply $\Delta_{\psi}^{i t} \in$ $\vee_{I} \mathscr{B}(I)$, and by the strong additivity of $\mathscr{B}$ we have $\Delta_{\psi}^{i t} \in \mathscr{B}(I) \vee \mathscr{B}\left(I^{\prime}\right)$ for all $t \in \mathbb{R}$. So $\Delta_{\psi}^{i t} \Delta^{-i t}$ commutes with $\Delta_{\psi}^{i t^{\prime}}$ for all $t, t^{\prime} \in \mathbb{R}$, hence $\Delta_{\psi}^{i t^{\prime}}$ commutes with $\Delta^{i t}$. It follows that for any $t^{\prime}, \operatorname{Ad}_{\Delta^{i t}}$ is a one-parameter automorphism of

$$
\operatorname{Ad}_{\Delta_{\psi}^{i t^{\prime}}}\left(\mathscr{B}(I) \vee \mathscr{A}\left(I_{1}\right)\right)
$$

preserving the vector state $(\cdot \Omega, \Omega)$. By the KMS condition [Strătilă 1981, p. 28] $\operatorname{Ad}_{\Delta^{i t}}$ is the modular automorphism of $\operatorname{Ad}_{\Delta_{\psi}^{i t^{\prime}}}\left(\mathscr{B}(I) \vee \mathscr{A}\left(I_{1}\right)\right)$ with respect to $\Omega$. Also $\overline{\mathscr{A}\left(I_{1}\right) \Omega}=\overline{\mathscr{A}(I) \Omega}$ for any $I$ by the Reeh-Schlieder Theorem. It follows by a theorem of Takesaki [Strătilă 1981, §9] that

$$
\operatorname{Ad}_{\Delta_{\psi}^{i t^{\prime}}}\left(\mathscr{B}(I) \vee \mathscr{A}\left(I_{1}\right)\right)=\mathscr{B}(I) \vee \mathscr{A}\left(I_{1}\right) \quad \text { for all } t^{\prime} \in \mathbb{R} .
$$

Since $\bigcup_{t^{\prime}} \Lambda_{I}\left(2 \pi t^{\prime}\right) I_{1}=I$, it follows that $\mathscr{B}(I) \vee \mathscr{A}\left(I_{1}\right)=\mathscr{A}(I)$, which proves the proposition since $\mathscr{B}$ is strongly additive.

Here is the key result of this section, a generalization of [Xu 1998, Theorem 3.3]:

Theorem 3.8. Let $\mathscr{B} \subset A$ be a strongly additive pair of conformal nets, and suppose $\mu, \lambda$ are representations of $\mathscr{B}$ localized on $I$.

(1) $x \in \mathscr{A}(I)$ satisfies $x \lambda(b)=\mu(b) x$ for all $b \in \mathscr{B}(I)$, then $x \alpha_{\lambda}(a)=\alpha_{\mu}(a) x$ for all $a \in \mathscr{A}(I)$.

(2) If $\mu, \lambda$ have finite index, then $\left\langle\alpha_{\mu}, \alpha_{\lambda}\right\rangle=\langle\mu \bar{\lambda}, \gamma\rangle$, where $\gamma$ is a representation of $\mathscr{B}$ unitarily equivalent to the defining representation of $\mathscr{B}$ on the vacuum Hilbert space of $\mathscr{A}$.

Proof. (1) Let $u_{1}$ (resp. $u_{2}$ ) be a unitary intertwiner in $\mathscr{B}(J)(J \supset I)$ transporting $\lambda$ (resp. $\mu$ ) to $\hat{\lambda}$ (resp. $\hat{\mu}$ ) localized on $I_{+}$. Since $x \lambda(a)=\mu(a) x$ for all $a \in \mathscr{B}(I)$ and $x \in \mathscr{A}(I)$, it follows by (3-2) in the proof of Proposition 3.1 that $u_{2} x u_{1}^{*} \in$ $\mathscr{A}(J) \cap \mathscr{B}(I)^{\prime}$. Choose $J$ so that $I \cup I_{+}=J$ minus one point. By the strong additive pair assumption, we have $\mathscr{A}\left(J^{\prime}\right) \cup \mathscr{B}(I)=\mathscr{A}\left(I_{+}^{\prime}\right)$, and so $\mathscr{A}(J) \cap \mathscr{B}(I)^{\prime}=\mathscr{A}\left(I_{+}\right)$. Thus $u_{2} x u_{1}^{*} \in \mathscr{A}\left(I_{+}\right)$, and (1) follows from (3-2) and the locality of the net $\mathscr{A}$. 
(2) Since $\lambda$ has finite index, one can find two isometries $R_{\lambda \bar{\lambda}} \in \operatorname{Hom}(\mathrm{id}, \lambda \bar{\lambda}$ ) and $R_{\bar{\lambda} \lambda} \in \operatorname{Hom}(\mathrm{id}, \bar{\lambda} \lambda)$ such that

$$
R_{\lambda \bar{\lambda}}^{*} \lambda\left(R_{\bar{\lambda} \lambda}\right)=R_{\bar{\lambda} \lambda}^{*} \bar{\lambda}\left(R_{\lambda \bar{\lambda}}\right)=\frac{1}{d(\lambda)} .
$$

Let $x \in \operatorname{Hom}\left(\alpha_{\lambda}, \alpha_{\mu}\right)$. Then $x R_{\lambda \bar{\lambda}} b=\mu \bar{\lambda}(b) x R_{\lambda \bar{\lambda}}$ for all $b \in \mathscr{B}_{B}(I)$. Note that $x R_{\lambda \bar{\lambda}} \in \mathscr{A}(I)$. Since $\mathscr{B}(I) \subset \mathscr{A}(I)$ is irreducible by Lemma 3.6(1), the vector space

$$
H_{\mu \bar{\lambda}}:=\{y \in \mathscr{A}(I) \mid y b=\mu \bar{\lambda}(b) y \text { for all } b \in \mathscr{B}(I)\}
$$

has finite dimension, equal to $\langle\mu \bar{\lambda}, \gamma\rangle$; see [Izumi et al. 1998, Theorem 3.3(i)]. Also, the map

$$
x \in \operatorname{Hom}\left(\alpha_{\lambda}, \alpha_{\mu}\right)_{\mathscr{A}} \mapsto x R_{\lambda \bar{\lambda}} \in H_{\mu \bar{\lambda}}
$$

is one-to-one because of the relations satisfied by $R_{\lambda \bar{\lambda}}, R_{\bar{\lambda} \lambda}$. Thus it is sufficient to show that the image the one-to-one map

$$
y \mapsto \mu\left(R_{\bar{\lambda} \lambda}^{*}\right) y, \quad \text { where } y \in H_{\mu \bar{\lambda}},
$$

is contained in $\operatorname{Hom}\left(\alpha_{\lambda}, \alpha_{\mu}\right)_{\mathscr{A}}$. But

$$
\mu\left(R_{\bar{\lambda} \lambda}^{*}\right) y \lambda(b)=\mu(b) \mu\left(R_{\bar{\lambda} \lambda}^{*}\right) y \quad \text { and } \quad \mu\left(R_{\bar{\lambda} \lambda}^{*}\right) y \in \mathscr{A}(I) .
$$

Thus, by part (1), $\mu\left(R_{\bar{\lambda} \lambda}^{*}\right) y \in \operatorname{Hom}\left(\alpha_{\lambda}, \alpha_{\mu}\right)_{\mathscr{A}}$.

Corollary 3.9. We keep the notation of Theorem 3.8 and assume that $\lambda$ has finite index.

(1) $\left[\alpha_{\bar{\lambda}}\right]=\left[\bar{\alpha}_{\lambda}\right]$.

(2) Let $H_{\lambda}:=\{x \in \mathscr{A}(I) \mid x b=\lambda(b) x$ for all $b \in \mathscr{B}(I)\}$. $H_{\lambda}$ is called the space of charged intertwiners associated with $\lambda$ [Longo and Rehren 1995]. Then $H_{\lambda}=\operatorname{Hom}\left(\mathrm{id}, \alpha_{\lambda}\right)$ and $\operatorname{dim} H_{\lambda}=\langle\gamma, \lambda\rangle \leq d_{\lambda}$.

(3) Let $\mathscr{A}_{f}(I) \subset \mathscr{A}$ be the subalgebra generated by $\mathscr{B}(I)$ and $H_{\lambda}, \lambda \in S$, where $S$ is a set of $(D H R)$ irreducible representations of $\mathscr{B}$ with finite statistical dimensions, and is closed under fusion and conjugation. Then $\mathscr{A}_{f}(I)$ is invariant as a set under the modular automorphism $\operatorname{Ad} U\left(\Lambda_{I}(t)\right.$ ) (Proposition 2.1), and

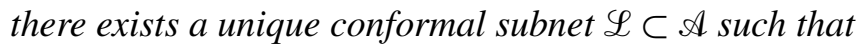

$$
\mathscr{B}(J) \subset \mathscr{L}_{f}(J) \subset \mathscr{A}(J) \quad \text { for all } J \in \mathscr{I}
$$

and $\mathscr{A}_{f}(I)=\mathscr{L}(I)$. Moreover, the vacuum representation $H_{\mathscr{L}}$ of the conformal net $\mathscr{L}$ as a representation of $\mathscr{B}$ decomposes as $H_{\mathscr{L}} \simeq \bigoplus_{\lambda \in S} \operatorname{dim} H_{\lambda} \lambda$.

Proof. (1) Thanks to Lemma 3.2 and Theorem 3.8(2), the proof from [Böckenhauer and Evans 1998] carries over:

$$
\left\langle\alpha_{\lambda}, \alpha_{\lambda}\right\rangle=\langle\lambda \bar{\lambda}, \gamma\rangle=\left\langle\alpha_{\lambda \bar{\lambda}}, \mathrm{id}\right\rangle=\left\langle\alpha_{\lambda} \alpha_{\bar{\lambda}}, \mathrm{id}\right\rangle=\left\langle\alpha_{\lambda}, \bar{\alpha}_{\bar{\lambda}}\right\rangle .
$$


Replacing $\lambda$ by $\bar{\lambda}$ we get $\left\langle\alpha_{\bar{\lambda}}, \alpha_{\bar{\lambda}}\right\rangle=\left\langle\alpha_{\bar{\lambda}}, \bar{\alpha}_{\lambda}\right\rangle$. Thus $\left\langle\alpha_{\bar{\lambda}}, \alpha_{\bar{\lambda}}\right\rangle=\left\langle\alpha_{\bar{\lambda}}, \bar{\alpha}_{\lambda}\right\rangle=\left\langle\bar{\alpha}_{\lambda}, \bar{\alpha}_{\lambda}\right\rangle$. Since $d_{\alpha_{\lambda}}$ and $d_{\alpha_{\bar{\lambda}}}$ are finite, we get the desired equality using Proposition 3.4.

(2) This follows directly from Theorem 3.8(2) and Proposition 3.4(1).

(3) Let $F_{\lambda}$ be the minimal conditional expectation, as on page 3. By [Longo 1992] we can choose a set of isometries $v_{i} \in \operatorname{Hom}\left(\mathrm{id}, \alpha_{\lambda}\right), 1 \leq i \leq \operatorname{dim} H_{\lambda}$ such that $F_{\lambda}\left(v_{i} v_{j}^{*}\right)=\left(1 / d_{\lambda}\right) \delta_{i j}$. Note that $E\left(v_{i} v_{j}^{*}\right) \in \mathscr{B}(I)^{\prime} \cap \mathscr{A}(I)=\mathbb{C}$, and by Section 3(3) we have

$$
E\left(v_{i} v_{j}^{*}\right)=F_{\lambda}\left(E\left(v_{i} v_{j}^{*}\right)\right)=E\left(F_{\lambda}\left(v_{i} v_{j}^{*}\right)\right)=\frac{1}{d_{\lambda}} \delta_{i j} .
$$

It follows that the operator $a_{\lambda}$ of [Izumi et al. 1998, p. 39] is the identity operator. (Our $\lambda$ corresponds to $\xi$ in that reference.) Then the argument in [Izumi et al. 1998, p. 41] shows that $\sigma_{t}^{\psi E}\left(v_{i}\right)=v_{i}$, where $\psi$ is a dominant weight on $\mathscr{B}(I)$ and $\sigma_{t}^{\psi E}$ is the modular automorphisms associated with the weight $\psi E$. By Haagerup's Theorem [Strătilă 1981, p. 156],

$$
\operatorname{Ad} U\left(\Lambda_{I}(t)\right)(\cdot)=\operatorname{Ad}_{u_{t}} \sigma_{t}^{\psi E}(\cdot)
$$

where $u_{t} \in \mathscr{B}(I)$. It follows that $\mathscr{A}_{f}(I)$ is invariant as a set under the modular automorphism $\operatorname{Ad} U\left(\Lambda_{I}(t)\right)$. Since $H_{\lambda}$ is finite-dimensional, the rest of the proof is the same as the proof in [Longo 2003, p. 18], as follows. $\Lambda_{I}(\mathbb{R})$ is the subgroup of $\mathbb{G}$ leaving $I$ globally fixed. For each $J \in \mathscr{F}$, set $\mathscr{L}(J)=\operatorname{Ad}_{U(g)}\left(\mathscr{A}_{f}(I)\right)$, where $g \in \mathbb{G}$ maps $I$ onto $J$. It is easy to check that $\mathscr{L}(J)$ is independent of the choice of $g$ as long as $g I=J$. Note that $\mathscr{L}(J)$ verifies locality since $\mathscr{L}(J) \subset \mathscr{A}(J)$. To show that $\mathscr{L}$ is a conformal net we just have to check the isotony property, namely $\mathscr{L}\left(J_{1}\right) \subset \mathscr{L}\left(J_{2}\right)$ if $J_{1} \subset J_{2}$. By conformal invariance we may assume that $J_{1}=I$ and that $J_{2}=g I$ for some $g \in \operatorname{PSL}(2, \mathbb{R})$, and it is sufficient to show that $\operatorname{Ad}_{U(g)}\left(\mathscr{A}_{f}(I)\right) \supset \mathscr{A}_{f}(I)$. Since $H_{\lambda}$ is finite-dimensional, by the second part of [Longo 2003, Corollary 19] we have $\operatorname{Ad}_{U(g)} H_{\lambda}=z_{\lambda}(g)^{*} H_{\lambda}$, where $z_{\lambda}(g) \in \mathscr{B}\left(J_{2}\right)$ is a unitary operator (compare [Longo 2003, (14)]). Hence

$$
\begin{aligned}
\operatorname{Ad}_{U(g)}\left(\mathscr{A}_{f}(I)\right) & =\left\{\operatorname{Ad}_{U(g)}(\mathscr{B}(I)), \operatorname{Ad}_{U(g)} H_{\lambda}, \lambda \in S\right\}^{\prime \prime} \\
& =\left\{\mathscr{B}\left(J_{2}\right), z_{\lambda}(g)^{*} H_{\lambda}, \lambda \in S\right\}^{\prime \prime} \\
& =\left\{\mathscr{B}\left(J_{2}\right), H_{\lambda}, \lambda \in S\right\}^{\prime \prime} \supset\left\{\mathscr{B}(I), H_{\lambda}, \lambda \in S\right\}^{\prime \prime}=\mathscr{A}_{f}(I) .
\end{aligned}
$$

Now let $\Omega$ be the vacuum vector for $\mathscr{A}$, since $\mathscr{L}$ is a conformal net, the vacuum representation space of $\mathscr{L}$ can be identified as $\overline{\mathscr{A}_{f}(I) \Omega}$ by Reeh-Schlieder theorem in Proposition 2.1. For each $\lambda \in S$, we choose isometries $v_{\lambda, i}\left(1 \leq i \leq \operatorname{dim} H_{\lambda}\right)$ as in the beginning of the proof of (3); we add a subscript $\lambda$ to emphasize the dependence on $\lambda$. Then the set consisting of $\sum_{\lambda \in S} v_{\lambda, i}^{*} x_{\lambda, i}, x_{\lambda, i} \in \mathscr{B}(I)$ where the sum is a finite sum is a dense subalgebra of $\mathscr{A}_{f}(I)$. Note that the space $X_{\lambda, i}:=$ 
$v_{\lambda, i}^{*} \overline{\mathscr{B}(I) \Omega}$ is invariant under the action of $\mathscr{B}$, and the restriction of $\mathscr{B}$ to this space is a representation of $\mathscr{B}$ unitarily equivalent to $\lambda$. Also note that $X_{\lambda, i} \perp X_{\lambda^{\prime}, i^{\prime}}$ if $(\lambda, i) \neq\left(\lambda^{\prime}, i^{\prime}\right)$ since $E\left(v_{\lambda, i} v_{\lambda^{\prime}, i^{\prime}}^{*}\right)=\left(1 / d_{\lambda}\right) \delta_{\lambda \lambda^{\prime}} \delta_{i i^{\prime}}$. Hence $\overline{\mathscr{A}_{f}(I) \Omega}$ is a direct sum of $X_{\lambda, i}$, and this proves the last part of (3).

The conformal net $\mathscr{L}$ constructed in Corollary 3.9(3) will be called the conformal subnet of $\mathscr{A}$ generated by $\mathscr{B}$ and the charged intertwiners associated with the set $S$.

\section{Applications}

Conformal nets with central charge 1. The irreducible representations of the Virasoro algebra with central charge 1 are classified as follows. For each $n \geq 0$, there is an irreducible representation with lowest weight $n$, which we denote by $L(1, n)$ following [Dong and Griess 1998]. Here 1 is the central charge. When $n=m^{2}$, with $2 m \in \mathbb{Z}$, the representation $L(1, n)$ is called degenerate due to the degeneracy of certain Verma modules. The vacuum representation is $L(1,0)$. All the $L(1, n)$ can be "exponentiated" to give irreducible projective representations of the group Diff $S^{1}$ of smooth diffeomorphisms of $S^{1}$; see [Goodman and Wallach 1984]. On the vacuum representation one can define a conformal net $\mathscr{A}_{c=1}$ as in [Gabbiani and Fröhlich 1993, §3], the so-called Virasoro net with central charge 1.

Let $\mathscr{A}_{\mathrm{SU}(2)_{1}}$ be the conformal net associated with loop group LSU(2) at level 1. The adjoint action of group $\mathrm{SO}(3)$ is proper (Definition 2.4) on $\mathscr{A}_{\mathrm{SU}(2)_{1}}$ and the fixed point is identified as $\mathscr{A}_{c=1}$, the Virasoro net with central charge $c=1$, in [Rehren 1994].

It follows from Theorem 2.6 that $\mathscr{A}_{c=1}$ is strongly additive. As pointed out in [Rehren 1994], the statistical dimension $d_{L\left(1, m^{2}\right)}$ of $L\left(1, m^{2}\right)$ is $2 m+1$ when $m$ is a nonnegative integer, and the fusion ring generated by $L\left(1, m^{2}\right)$ are the same as representation rings of $\mathrm{SO}(3)$. The following lemma generalizes this to the case when $2 m$ is a nonnegative integer:

Lemma 4.1. Assume that $2 m$ is a nonnegative integer. Then $d_{L\left(1, m^{2}\right)}=2 m+1$ and the fusion ring generated by $L\left(1, m^{2}\right)$ is isomorphic to the representation ring of $\mathrm{SU}(2)$.

Proof. Consider the conformal inclusion

$$
\mathrm{LSU}(2)_{1} \times \mathrm{LU}(1)_{2} \subset \mathrm{LU}(2)_{1}
$$

(compare [Xu 2000, §3.1]). The group $\mathrm{SU}(2)$ acts properly on the net $\mathscr{A}_{U(2)_{1}}$ with fixed point net $\mathscr{A}_{c=1} \times \mathscr{A}_{U(1)_{2}}$. The net $\mathscr{A}_{U(2)_{1}}$ is not local, but satisfies twisted duality [Wassermann 1998, §15]. So [Doplicher and Roberts 1990, Theorem 3.6] applies in this case, and the fusion of those irreducible representations of $\mathscr{A}_{c=1} \times \mathscr{A}_{U(1)_{2}}$ appearing in $\mathscr{A}_{U(2)_{1}}$ is given by the fusion ring of finite-dimensional 
representations of $S U(2)$. Since irreducible covariant representations of $\mathscr{A}_{U(1)_{2}}$ have statistical dimension equal to 1 and generate an abelian group $\mathbb{Z}_{2}$ under the fusion, the lemma follows.

Let $\mathscr{A}$ be a conformal net. Following [Kawahigashi and Longo 2004], we say that $\mathscr{A}$ is a diffeomorphism covariant net if there exists a unitary projective representation $U$ of Diff $S^{1}$ on $H$ extending the unitary representation of $\operatorname{PSL}(2, \mathbb{R})$ and such that

$$
U(g) \mathscr{A}(I) U\left(g^{*}\right)=\mathscr{A}(g I) \quad \text { for } g \in \operatorname{Diff} S^{1}, I \in \mathscr{I} .
$$

We say that $\mathscr{A}$ is a conformal net with central charge 1 if $\mathscr{A}$ is a diffeomorphism covariant net containing $\mathscr{A}_{c=1}$ as a conformal subnet, such that

$$
U(\operatorname{Diff} I)^{\prime \prime}=\left(\mathscr{A}_{c=1}\right)(I) \quad \text { for all } I \in \mathscr{I},
$$

where Diff $I$ denotes the group of smooth diffeomorphisms $g$ of $S^{1}$ satisfying $g(t)=t, t \in I^{\prime}$.

We describe the known list of such nets. Let $G$ be a closed group of $\operatorname{SO}(3)$. Such groups are well known to be of A-D-E groups corresponding to affine A-D-E graphs. Let $\hat{G}$ be twofold covering group of $G$ in SU(2). The Perron-Frobenius eigenvectors given in [Goodman et al. 1989, p. 14] are the dimensions of the irreducible representations of the twofold covering group $\hat{G}$. Since $\mathscr{A}_{c=1}$ can be identified with $\mathscr{A}_{\mathrm{SU}(2)_{1}}^{\mathrm{SO}}, \mathscr{A}_{\mathrm{SU}()_{1}}^{G}$ is a conformal net with central charge $c=1$. The remaining two cases are $\mathscr{A}_{U(1)_{2 n}}$ and its $\mathbb{Z}_{2}$ orbifold $\mathscr{A}_{U(1)_{2 n}}^{\mathbb{Z}_{2}}$ as studied in [Xu 2001], where $n$ is not the square of an integer. So the known list of conformal nets with central charge 1 is:

$$
\mathscr{A}_{\mathrm{SU}(2) 1}^{G}, \mathscr{A}_{U(1)_{2 n}}, \mathscr{A}_{U(1)_{2 n}}^{\mathbb{Z}_{2}}
$$

where $G$ is a closed subgroup of $\mathrm{SO}(3)$ and $n$ is not the square of an integer.

It has been conjectured [Dijkgraaf et al. 1989] that this list exhausts all conformal theories with central charge 1 .

When $G$ is a finite group, $\mathscr{A}_{\mathrm{SU}(2)_{1}}^{G}$ is completely rational by Proposition 2.3.

$\mathscr{A}_{U(1)_{2 n}}$ and $\mathscr{A}_{U(1)_{2 n}}^{\mathbb{Z}_{2}}$ are also completely rational and all irreducible representations are obtained in [Xu 2001]. The irreducible representations of $\mathscr{A}_{U(1)_{2 n}}$ will be denoted by $\pi_{i}$, for $i \in \mathbb{Z}_{2 n}$. They generate a fusion ring isomorphic to $\mathbb{Z}_{2 n}$.

When $G=U(1), A_{\mathrm{SU}(2)_{1}}^{G}$ is the net corresponding to the Heisenberg group $H(1)$, defined as the set $C^{\infty}\left(S^{1}, \mathbb{R}\right) \times S^{1}$ with the multiplication

$$
\left(f_{1}, x_{1}\right) \cdot\left(f_{2}, x_{2}\right)=\left(f_{1}+f_{2}, e^{\int_{S^{1}} f_{1} f_{2}^{\prime}} x_{1} x_{2}\right)
$$

(see [Pressley and Segal 1986, §9.5]). Note that $(x, 1)$, where $x \in \mathbb{R}$ is regarded as a constant map, is in the center of $H(1)$. For each real number $q$, there is an irreducible representation of $H(1)$ denoted by $F_{q}$, where $(x, 1)$ acts on $F_{q}$ as 
$(x, 1) \mapsto q x$, and these are all the irreducible representations of $H(1)$ [Pressley and Segal 1986, Proposition 9.5.10]. The net $\mathscr{A}_{\mathrm{SU}(2)_{1}}^{U(1)}$ is related to $H(1)$ as follows. $F_{0}$ is the vacuum representation of $\mathscr{A}_{\mathrm{SU}(2)_{1}}^{U(1)}$, and

$$
\mathscr{A}_{\mathrm{SU}(2)_{1}}^{U(1)}(I)=\pi_{F_{0}}\left(C_{0}^{\infty}(I, \mathbb{R})\right)^{\prime \prime},
$$

where $C_{0}^{\infty}(I, \mathbb{R})$ is the set of smooth maps from $I$ to $\mathbb{R}$ that vanish on the boundary, and is considered as a subspace of $C^{\infty}\left(S^{1}, \mathbb{R}\right)$. Each $F_{q}$ is also an irreducible representation of $\mathscr{A}_{\mathrm{SU}(2)_{1}}^{U(1)}$. The net $\mathscr{A}_{\mathrm{SU}(2),}^{U(1)}$ was studied in [Buchholz et al. 1988].

Some decompositions of the vacuum representation of the nets in the list above when restricting to $\mathscr{A}_{c=1}$ are also known (see [Dong and Griess 1998, Proposition 2.2, Theorems 2.7 and 2.9], where $n$ corresponds to our $2 n$ ). They are:

If $n$ is not the square of an integer, then

$$
\begin{aligned}
& H_{\mathscr{A}_{U(1)_{2 n}}}=\bigoplus_{p \geq 0} L\left(1, p^{2}\right) \oplus \bigoplus_{m>0} 2 L\left(1, m^{2} n\right), \\
& H_{\mathscr{A}_{U(1)_{2 n}}^{\mathbb{Z}_{2}}}=\bigoplus_{p \geq 0} L\left(1,4 p^{2}\right) \oplus \bigoplus_{m>0} L\left(1, m^{2} n\right) .
\end{aligned}
$$

If $n=k^{2}$, where $k$ is a nonnegative integer, then

$$
H_{\mathscr{A}_{U(1) 2 n}}=\bigoplus_{m \geq 0} \bigoplus_{0 \leq p \leq k-1}(2 m+1) L\left(1,(m k+p)^{2}\right)
$$

When $G=U(1)$ or $G=D_{\infty}$, the infinite dihedral group, we have

$$
\begin{aligned}
& H_{\mathscr{A}_{\mathrm{SU}(2) 1}^{U(1)}}=\bigoplus_{p \geq 0} L\left(1, p^{2}\right), \\
& H_{\mathscr{A}_{\mathrm{SU}(2) 1}^{D \infty}}=\bigoplus_{p \geq 0} L\left(1,4 p^{2}\right) .
\end{aligned}
$$

Recall that $F_{q}$ is the irreducible representation of $\mathscr{A}_{\mathrm{SU}(2))_{1}}^{U(1)}$ corresponding to an irreducible representation of $H(1)$ labeled by a real number $q$. The decompositions of $F_{q}$ with respect to $\mathscr{A}_{c=1}$ is also well known [Dong and Griess 1998]:

If $q=\frac{1}{4} p^{2}$ for some nonnegative integer $p$, then

$$
F_{q}=\bigoplus_{-p / 2 \leq m \leq p / 2, m+p / 2 \in \mathbb{Z}} L\left(1, m^{2}\right)
$$

If $4 q$ is not the square of an integer, then $F_{q}=L(1, \sqrt{q})$.

By definition, if $\mathscr{A}$ is a conformal net with central charge 1, the pair $\mathscr{A}_{c=1} \subset \mathscr{A}$ satisfies the condition of Proposition 3.7, and hence is a strongly additive pair. By Lemma 4.1, the principal graph of $L\left(1, \frac{1}{4}\right)$ is $A_{\infty}$, and $d_{L(1,1 / 4)}=2$. It follows from Proposition 3.4 that $\alpha_{L\left(1, p^{2}\right)}=d_{L\left(1, p^{2}\right)}=2 p+1$ for all nonnegative integer $2 p$. Since $\alpha_{f}:=\alpha_{L(1,1 / 4)}$ has minimal index 4, its principal graph are determined in [Goodman et al. 1989; Popa 1994]. We now list some properties of $\alpha_{f}$ :

Lemma 4.2. The possible principal graphs of $\alpha_{f}$ are the A-D-E graphs of [Goodman et al. 1989, p. 19]. More precisely: 
(1) $\left[\alpha_{f}\right]=\left[\bar{\alpha}_{f}\right]$;

(2) If $\alpha_{f}$ is irreducible, its principal graph is one of: the D, E graphs of [Goodman et al. 1989, p. 19] or $A_{\infty}$ and $D_{\infty}$ in [Goodman et al. 1989, p. 217].

(3) If $\left\langle\alpha_{f}, \alpha_{f}\right\rangle=4$, its principal graph is $A_{1}^{(1)}$.

(4) If $\left\langle\alpha_{f}, \alpha_{f}\right\rangle=2$, the principal graph is either $A_{\infty, \infty}$ or $A_{n}^{(1)}$.

Proof. (1) follows from Corollary 3.9, and (2)-(4) from [Popa 1995, §4].

As a warmup exercise, we work out $\alpha_{f}$ for the list (4-1). We first prove a lemma that will be used later:

Lemma 4.3. Every covariant representation of $\mathscr{A}_{\mathrm{SU}(2)_{1}}^{U(1)}$ is a direct sum of irreducible representations, and every irreducible covariant representation of $\mathscr{A}_{\mathrm{SU}(2)_{1}}^{U(1)}$ is isomorphic to some $F_{q}$.

Proof. Let $\pi$ be a covariant representation of $\mathscr{A}_{\mathrm{SU}(2)_{1}}^{U(1)}$. Recall from (4-2) above the product rule in $H(1)$. We will write $\left(f_{i}, 1\right)$ simply as $f_{i}$.

Let $I_{i} \in \mathscr{I}, 1 \leq i \leq n$, be an open covering of $S^{1}$, with a partition of unity $\phi_{i}$ subordinate to it $\left(\operatorname{so} \operatorname{supp}\left(\phi_{i}\right) \subset I_{i}\right.$ for each $\left.i\right)$. If $f: S^{1} \rightarrow \mathbb{R}$, we assume that

$$
f=\prod_{k}\left(f \phi_{k}, 1\right) C(f, \phi) \in H(1),
$$

where $C(f, \phi) \in \mathbb{C}$ is a phase coming from the product rules. We have $\pi_{F_{0}}\left(f \phi_{k}\right) \in$ $\mathscr{A}_{\mathrm{SU}(2)_{1}}^{U(1)}\left(I_{k}\right)$, and we define

$$
\pi(f):=\prod_{k} \pi_{I_{k}}\left(\pi_{F_{0}}\left(f \phi_{k}\right)\right) C(f, \phi)
$$

It is routine to check that $\pi(f)$ is independent of the choices of open covering and partition of unity (use the product rules in $H(1)$ and isotony), and that it gives a representation of $H(1)$ with positive energy. The lemma now follows from [Pressley and Segal 1986, Proposition 9.5.10] and its proof.

Lemma 4.4. (1) If $\mathscr{A}=\mathscr{A}_{c=1}$, the principal graph of $\alpha_{f}$ is $A_{\infty}$.

(2) If $\mathscr{A}=\mathscr{A}_{U(1)_{2 k^{2}}}$, the principal graph of $\alpha_{f}$ is $A_{2 k-1}^{(1)}$.

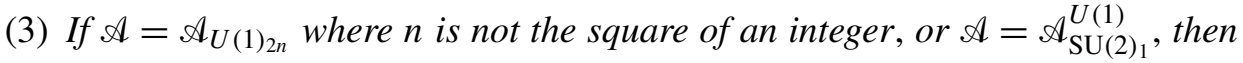
the principal graph of $\alpha_{f}$ is $A_{\infty,-\infty}$.

(4) If $\mathscr{A}=\mathscr{A}_{U(1)_{2 k^{2}}}^{\mathbb{Z}_{2}}$, the principal graph of $\alpha_{f}$ is $D_{k}^{(1)}$.

(5) If $\mathscr{A}=\mathscr{A}_{U(1)_{2 n}}$ where $n$ is not the square of an integer, or $\mathscr{A}=\mathscr{A}_{\mathrm{SU}(2)_{1}}^{D_{\infty}}$, then the principal graph of $\alpha_{f}$ is $D_{\infty,-\infty}$.

(6) If $\mathscr{A}=\mathscr{A}_{\mathrm{SU}(2)_{1}}^{E_{i}}$ for $i=6,7,8$, the principal graph of $\alpha_{f}$ is $E_{i}$. 
Proof. (1) follows from Lemma 4.1. If $\mathscr{A}=\mathscr{A}_{U(1)_{2 k^{2}}}$, it follows from the branching rules, Lemma 4.1 and Proposition 3.1 that $\alpha_{f}$ is localized on $I$, and by Theorem 3.8(2) $\left\langle\alpha_{f}, \alpha_{f}\right\rangle=4$ when $k=1$ and $\left\langle\alpha_{f}, \alpha_{f}\right\rangle=2$ when $k>1$. So (2) is proved for $k=1$ by Lemma 4.2. Note that when $k=1, \mathscr{A}_{U(1)_{2}}$ can be identified with $\mathscr{A}_{\mathrm{SU}(2)_{1}}$, and it is easy to check that $\left[\alpha_{f}\right]=2[\tau]$, where $\tau$ is the irreducible representation of $\mathscr{A}_{\mathrm{SU}(2)_{1}}$ which is not the vacuum representation and [ $\left.\tau\right]^{2}=[1]$.

When $k>1$ we have $\left[\alpha_{f}\right]=\left[\sigma_{1}\right]+\left[\sigma_{2}\right]$, where $\sigma_{1}, \sigma_{2}$ are representations of $\mathscr{A}_{U(1)_{2 k^{2}}}$ which have been classified, and by (1) of Lemma 3.3 we must have $\left[\alpha_{f}\right]=$ $\left[\pi_{k}\right]+\left[\pi_{-k}\right]$ and (2) follows. If $\mathscr{A}=\mathscr{A}_{U(1)_{2 n}}$, where $n$ is not the square of an integer, or $\mathscr{A}=\mathscr{A}_{\mathrm{SU}(2)_{1}}^{U(1)}$, it follows from the branching rules, Lemma 4.1 and Theorem 3.8(2) that $\left\langle\alpha_{L\left(1, m^{2}\right)}, \mathrm{id}\right\rangle=1,\left\langle\alpha_{L\left(1, m^{2}\right)}, \alpha_{L\left(1, m^{2}\right)}\right\rangle=2 m+1$ for all $m \in \mathbb{N}$, and $\left\langle\alpha_{f}, \alpha_{f}\right\rangle=2$. So $\left[\alpha_{f}\right]=\left[\sigma_{1}\right]+\left[\sigma_{2}\right]$ and $d_{\sigma_{1}}=d_{\sigma_{2}}=1$. By Lemma 4.2, then, $\left[\alpha_{f}\right]=\left[\bar{\alpha}_{f}\right]$, so either $\left[\bar{\sigma}_{1}\right]=\left[\sigma_{2}\right]$ or $\left[\bar{\sigma}_{i}\right]=\left[\sigma_{i}\right]$ for $i=1,2$. If the latter, then $\left[\sigma_{1}^{2}\right]=\left[\sigma_{2}^{2}\right]=[1]$ and

$$
\left[\alpha_{L(1,1)}\right]=\left[\alpha_{f}^{2}\right]-[1]=\left[\sigma_{1} \sigma_{2}\right]+\left[\sigma_{1} \sigma_{2}\right]+[1] .
$$

By Proposition 3.5(1),

$$
\left[\sigma_{1} \alpha_{f}\right]=\left[\sigma_{1} \sigma_{2}\right]+[1]=\left[\alpha_{f} \sigma_{1}\right]=\left[\sigma_{2} \sigma_{1}\right]+[1] .
$$

Hence $\left[\sigma_{1} \sigma_{2}\right]=\left[\sigma_{2} \sigma_{1}\right]$, implying that $\left\langle\alpha_{L(1,1)}, \alpha_{L(1,1)}\right\rangle=5$, which contradicts $\left\langle\alpha_{L(1,1)}, \alpha_{L(1,1)}\right\rangle=3$. So we have $\left[\alpha_{f}\right]=\left[\sigma_{1}\right]+\left[\sigma_{2}\right],\left[\bar{\sigma}_{1}\right]=\left[\sigma_{2}\right]$, and it follows that

$$
\left[\alpha_{L\left(1, m^{2}\right)}\right]=\sum_{-m \leq k \leq m}\left[\sigma_{1}^{2 k}\right]
$$

Since $\left\langle\alpha_{L\left(1, m^{2}\right)}, 1\right\rangle=1$, it follows that $\left[\sigma_{1}^{k}\right] \neq[1]$ for all $k$, and the principal graph of $\alpha_{f}$ is $A_{\infty,-\infty}$.

When $\mathscr{A}=\mathscr{A}_{U(1)_{2 k^{2}}}$ or $\mathscr{A}=\mathscr{A}_{\mathrm{SU}(2)_{1}}^{E_{i}}$ for $i=6,7,8, \alpha_{f}$ is irreducible and a representation of $\mathscr{A}$, denoted by $f_{1}$. We will call this representation the vector representation. Since $\mathscr{A}$ is completely rational, it follows that the graph of $\alpha_{f}$ must be finite and hence must be finite $D, E$ type. Consider the inclusions of conformal nets

$$
\mathscr{A}_{c=1} \subset \mathscr{A} \subset \mathscr{A}_{\mathrm{SU}(2)_{1}},
$$

and denote by $\alpha_{f_{1}}$ the induction of $f_{1}$ (as a local representation of $\mathscr{A}$ ) from $\mathscr{A}$ to $A_{\mathrm{SU}(2)_{1}}$; by Lemma 3.3(3),

$$
\alpha_{f_{1}}=\alpha_{L(1,1 / 4)}^{\mathscr{A}_{c=1} \rightarrow A_{\mathrm{SU}(2) 1}} .
$$

This equality and the preceding proof imply that $\left[\alpha_{f_{1}}\right]=2[\tau]$, where $\tau$ is the irreducible representation of $\mathscr{A}_{\mathrm{SU}(2)_{1}}$ which is not the vacuum representation and $[\tau]^{2}=[1]$. 
For any irreducible representation $\lambda \prec f_{1}^{2 n}$ with $n \in \mathbb{N}$, set $m_{\lambda}:=\left\langle\alpha_{\lambda}, 1\right\rangle$, Then $m_{\lambda}=d_{\lambda}$, and by Theorem 3.8(2), $m_{\lambda}$ is the multiplicity of $\lambda$ appearing in the vacuum representation of $\mathscr{A}_{\mathrm{SU}(2)_{1}}$, and is equal to the dimension of the representation of the corresponding D-E groups since $\mathscr{A}$ is the fixed point net of $\mathscr{A}_{\mathrm{SU}(2))_{1}}$ under the action of such groups. Note that the Perron-Frobenius eigenvectors listed on [Goodman et al. 1989, p. 14] are the statistical dimensions associated with the corresponding representations. Hence the graph is uniquely determined by the corresponding groups to be those of (4) and (6).

(5) follows by inspecting the branching rules and using Theorem 3.8 as we have done in proving (1)-(3).

As a byproduct of this proof, we have the following proposition, which contains the main result of [Rehren and Tuneke 2000]:

Proposition 4.5. Let $2 m$ be a nonnegative integer, and $n \geq 0$. Then

$$
\left[L\left(1, m^{2}\right)\right][L(1, n)]=\sum_{\substack{-m \leq k \leq m \\ k+m \in \mathbb{Z}}}\left[L\left(1,(k+\sqrt{n})^{2}\right] .\right.
$$

Proof. By Lemma 4.1 it is sufficient to consider the case when $L(1, n)$ is generic, i.e., when $4 n$ is not the square of an integer. Now, the inclusion $\mathscr{A}_{c=1} \subset \mathscr{A}_{\mathrm{SU}(2)_{1}}^{U(1)}$ is a strongly additive pair. As in the proof of Lemma 4.4(3), by the branching rules, Lemma 4.1 and Proposition 3.1, $\alpha_{f}$ is a (DHR) representation of $\mathscr{A}_{\mathrm{SU}(2)_{1}}^{U(1)}$, and $\left[\alpha_{f}\right]=\left[\sigma_{1}\right]+\left[\sigma_{2}\right],\left[\bar{\sigma}_{1}\right]=\left[\sigma_{2}\right]$. By Lemma 4.3, $\left[\sigma_{1}\right]=\left[F_{q}\right]$ for some real $q$, and since by Lemma 3.3(1)

$$
\left\langle\alpha_{f}, F_{q}\right\rangle \leq\left\langle L(1,1 / 4), \gamma F_{q}\right\rangle,
$$

inspecting the branching rules we conclude that $\left[\alpha_{L(1,1 / 4)}\right]=\left[F_{1 / 2}\right]+\left[F_{-1 / 2}\right]$. It follows by Lemma 4.1 that

$$
\left[\alpha_{L\left(1, m^{2}\right)}\right]=\sum_{\substack{-m \leq k \leq m \\ k+m \in \mathbb{Z}}}\left[F_{k}\right]
$$

Note that $\left[\gamma\left(F_{\sqrt{n}}\right)\right]=[L(1, n)]$ by the branching rules and [Longo and Rehren 1995, Proposition 3.1]. We have:

$$
\begin{aligned}
{\left[L\left(1, m^{2}\right)\right][L(1, n)] } & =\left[L\left(1, m^{2}\right)\right]\left[\gamma F_{\sqrt{n}}\right]=\left[\gamma \alpha_{L\left(1, m^{2}\right)} F_{\sqrt{n}}\right] \\
& =[\gamma] \sum_{\substack{-m \leq k \leq m \\
k+m \in \mathbb{Z}}}\left[F_{k} F_{\sqrt{n}}\right] \\
& =[\gamma] \sum_{\substack{-m \leq k \leq m \\
k+m \in \mathbb{Z}}}\left[F_{k+\sqrt{n}}\right]=\sum_{\substack{-m \leq k \leq m \\
k+m \in \mathbb{Z}}}\left[L_{(k+\sqrt{n})^{2}}\right] .
\end{aligned}
$$


Classifications. As in [Kawahigashi and Longo 2004], two conformal nets $\mathscr{A}$ and $\mathscr{B}$ are said to be isomorphic, denoted by $\mathscr{A} \simeq \mathscr{B}$, if there is a unitary operator $U: \mathscr{H}_{\mathscr{A}} \rightarrow \mathscr{H}_{\mathscr{B}}$ such that $U^{*} \mathscr{B}(J) U=\mathscr{A}(J)$ for all $J \in \mathscr{I}$ and that $U \Omega_{\mathscr{A}}=\Omega_{\mathscr{B}}$, where $\Omega_{\mathscr{A}}$ and $\Omega_{\mathscr{B}}$ are the vacuum vectors.

By the proof of Lemma 4.4, we can infer the type of principal graphs for $\alpha_{f}$ from branching rules and $\gamma$ from Theorem 3.8. Also thanks to Theorem 3.8, we can deduce information about $\gamma$ from the type of principal graph of $\alpha_{f}$. This is the basic strategy that we follow to classify conformal nets with central charge $c=1$. This will work out under the following spectrum condition:

Spectrum Condition. A conformal net with central charge $c=1$ satisfies the spectrum condition if a degenerate representation of the Virasoro net other than the vacuum representation must appear in the vacuum representation of $\mathscr{A}$ if $\mathscr{A} \neq \mathscr{A}_{c=1}$.

Theorem 4.6. If a conformal net $\mathscr{A}$ with central charge $c=1$ satisfies the spectrum condition, then $A$ is isomorphic to one of the nets on the list (4-1).

The proof is divided into the following steps:

4.6.1. Discrete case: Full spectrum. In this section we assume that

$$
\gamma=\bigoplus_{0 \leq m}(2 m+1) L\left(1, m^{2}\right) .
$$

We'd like to show that $\mathscr{A} \simeq \mathscr{A}_{\mathrm{SU}(2)_{1}}$. This is essentially an application of reconstruction theorem of Doplicher and Roberts [1989; 1990]. We give a sketch of the proof and refer to those papers for details. First by our assumption we can assume that $\mathscr{A}$ and $\mathscr{A}_{\mathrm{SU}(2)_{1}}$ acting on the same Hilbert space, and $\mathscr{A}_{c=1}$ is a common conformal subnet. Let $\Delta:=\left\{L\left(1, p^{2}\right), p \in \mathbb{Z}\right\}$. We note that $L(1,1)$ has permutation symmetry and satisfies special conjugate property, and $\Delta$ is generated by $L(1,1)$, and is specially directed as defined on [Doplicher and Roberts 1989, p. 98]. Fix an interval $I$. Choose charged intertwiners $\psi^{\mathscr{A}} \in \mathscr{A}(I), \psi \in \mathscr{A}_{\mathrm{SU}(2)_{1}}(I)$ for the set $\Delta$ (it is enough to choose charged intertwiners for $L(1,1)$ ). Denote by $u_{\mathscr{A}}$ the $C^{*}$ algebra generated by $\boldsymbol{U}_{\mathscr{A}_{c=1}}$ and $\psi^{\mathcal{A}}$, and by $\mathcal{U}$ the one generated by $\boldsymbol{u}_{\mathscr{A}_{c=1}}$ and $\psi$. Note that $\mathcal{u}_{\mathscr{A}} \cap u_{\mathscr{A}_{c=1}^{\prime}}^{\prime}=\mathbb{C}$. By [Doplicher and Roberts 1989, p. 93-94] there exists an epimorphism $\phi: \mathcal{U} \rightarrow \boldsymbol{U}_{\mathscr{A}}$ such that $\phi=\mathrm{id}$ on $\boldsymbol{U}_{\mathscr{A}_{c=1}}$. Using $\phi$ one can define an action of $\mathrm{SO}(3)$ which commutes with $\boldsymbol{U}_{\unlhd_{c=1}}$. One checks that $\phi$ commutes with the adjoint action of $\mathrm{SO}(3)$, and so $\operatorname{ker} \phi$ is $\mathrm{SO}(3)$ invariant. Then the argument on p. 95 of the same reference shows that $\operatorname{ker} \phi=\{0\}$, and so $\phi$ is an isomorphism. Now define a unitary operator $U$ on $H$ by $U m \Omega=\phi(m) \Omega$ where $\Omega$ is the vacuum vector. Then

$$
U \mathscr{u} U^{*}=u_{\mathscr{A}}
$$

and $U$ commutes with $\mho_{\mathscr{A}_{c=1}}$. Passing to the von Neumann algebra generated by $U$ and $\varkappa_{\mathscr{A}}$, we have $U \mathscr{A}(I) U^{*}=\mathscr{A}_{\mathrm{SU}(2)_{1}}(I), U \Omega=\Omega$. Since $U$ commutes with 
$\mathcal{U}_{\mathscr{A}_{c=1}}, U$ commutes with PSL(2, R) by the strong additivity of $\mathscr{A}_{c=1}$, and it follows that

$$
U \mathscr{A}(J) U^{*}=\mathscr{A}_{\mathrm{SU}(2)_{1}}(J) \text { for all } J \in \mathscr{I}, U \Omega=\Omega .
$$

4.6.2. General discrete case. In this section we assume that

$$
\gamma=\bigoplus_{p \geq 0} m_{p} L\left(1, p^{2}\right) \text {. }
$$

Here $m_{p} \leq 2 p+1$ by Corollary 3.9(2). By Lemma 4.1 and Proposition 3.1, $\alpha_{L\left(1, p^{2}\right)}$ is localized. Consider the set of representations $\Delta:\left\{\lambda \prec \alpha_{L(1,1)}^{n}, n \in \mathbb{N}\right\}$ of $\mathscr{A}$. Since $\epsilon\left(\alpha_{L(1,1)}, \alpha_{L(1,1)}\right)=\epsilon(L(1,1), L(1,1))$ by Lemma 3.3(2), the set $\Delta$ has permutation symmetry, and $\mathscr{A}$ is strongly additive, we can apply Doplicher-Roberts reconstruction as in [Müger 1999, Proposition 3.9] to obtain conformal net $\mathscr{C}$ such that $\mathscr{A}_{c=1} \subset \mathscr{A} \subset \mathscr{C}$. Consider the conformal nets $\mathscr{A}_{c=1} \subset \mathscr{C}$. We claim that $m_{p}^{\mathscr{C}}=2 p+1$ in this case, where $m_{p}^{\mathscr{C}}$ is the multiplicity of $L\left(1, p^{2}\right)$ which appears in the vacuum representation of $\mathscr{C}$. We have $m_{p}^{\mathscr{C}} \geq \sum_{\lambda}\left\langle\alpha_{L\left(1, p^{2}\right)}, \lambda\right\rangle$, where the sum is over those irreducible representations $\lambda$ of $\mathscr{A}$ which appears in the vacuum representation of $\mathscr{b}$. We note that $\sum_{\lambda}\left\langle\alpha_{L\left(1, p^{2}\right)}, \lambda\right\rangle$ is completely determined by the principal graph of $\alpha_{f}$, which corresponds to A-D-E groups. So the number $\sum_{\lambda}\left\langle\alpha_{L\left(1, p^{2}\right)}, \lambda\right\rangle$ depends only the type of A-D-E graph associated with $\alpha_{f}$. Since all types of such graphs have appeared in Lemma 4.4, and in each case, it is easy to check the number $\sum_{\lambda}\left\langle\alpha_{L\left(1, p^{2}\right)}, \lambda\right\rangle$ is $2 p+1$, since for $\mathscr{A}_{c=1} \subset \mathscr{A}_{\mathrm{SU}(2))_{1}}^{G}$, where $G$ is a closed subgroup of $\mathrm{SO}(3)$, the above reconstruction give us $\mathscr{A}_{\mathrm{SU}(2)_{1}}$. It follows that $m_{p}^{\mathscr{C}} \geq 2 p+1$, and then that $m_{p}^{\mathscr{C}}=2 p+1$ by Corollary 3.9(2). Now consider the conformal subnet $\mathscr{D} \subset \mathscr{C}$ generated by $\mathscr{A}_{c=1}$ and the charged intertwiners for $\left\{L\left(1, p^{2}\right): p \geq 0\right\}$ whose existence is guaranteed by the same corollary. Now $\mathscr{D}$ has full spectrum as in $\S 4.6 .1$, and so $\mathscr{A}_{c=1} \subset \mathscr{A} \subset \mathscr{D} \simeq \mathscr{A}_{\mathrm{SU}(2)_{1}}$.

Since $\mathscr{A}_{c=1}$ is the fixed point net of $\mathscr{A}_{\mathrm{SU}(2)_{1}}$ under the action of $\mathrm{SO}(3)$, by [Izumi et al. 1998] there exists a closed subgroup $G_{1}$ of $\operatorname{SO}(3)$ such that $\mathscr{A}(I)$ is the fixedpoint subalgebra of $\mathscr{A}_{\mathrm{SU}(2)_{1}}(I)$ under the action of $G_{1}$. Since $G_{1}$ commutes with $\operatorname{PSL}(2, \mathbb{R}), \mathscr{A}$ is the fixed point net of $\mathscr{A}_{\mathrm{SU}(2)_{1}}$ under the action of $G_{1}$. Since $G_{1}$ is classified as one of the A-D-E groups, it follows that $\mathscr{A}$ is in the list (4-1).

4.6.3. The cases $\mathscr{A}_{c=1} \subset \mathscr{A}_{U(1)_{2 n}} \subset \mathscr{A}$ and $\mathscr{A}_{c=1} \subset \mathscr{A}_{\mathrm{SU}(2)_{1}}^{U(1)} \subset \mathscr{A}$. We first consider the case $\mathscr{A}_{c=1} \subset \mathscr{A}_{U(1)_{2 n}} \subset \mathscr{A}$.

Recall that $\mathscr{A}_{U(1)_{2 n}}$ is completely rational and that its representations are labeled by $\pi_{i}$, for $0 \leq i \leq 2 n-1$; they have conformal dimensions $i^{2} /\left(4 n^{2}\right)+\mathbb{N}$ (these are the eigenvalues of the action of rotations) and statistical dimension 1. The fusion ring generated by $\pi_{i}$ is $\mathbb{Z}_{2 n}$. Since $\mathscr{A}_{U(1)_{2 n}} \subset \mathscr{A}$ is a strongly additive pair by Lemma 3.6(3), the vacuum representation $H_{\mathscr{A}}$ decomposes into a representation of $\mathscr{A}_{U(1)_{2 n}}$ as $H_{\mathscr{A}}=\oplus_{i} m_{i} \pi_{i}$ with $m_{i} \leq 1$ by Corollary 3.9(2). Also if $m_{i}=1$, again by the same corollary we have $m_{i}=1=\left\langle 1, \alpha_{i}\right\rangle$, hence $\left[\alpha_{i}\right]=[1]$ since $d_{\alpha_{i}}=d_{i}=1$. So 
the set of $H_{i}$ appearing in the decompositions of $H_{\mathscr{A}}$ is an abelian subgroup of $\mathbb{Z}_{2 n}$. Let $i_{0}>0$ be the generator of this abelian subgroup. There is a positive integer $k$ such that $k i_{0}=2 n$. On the other hand, we must have $i_{0}^{2} /\left(4 n^{2}\right) \in \mathbb{Z}$, and we assume that $k_{0}$ is a positive integer such that $i_{0}^{2}=4 n k_{0}$. It follows that $n=k^{2} k_{0}$. Now we compare the inclusions

$$
\mathscr{A}_{U(1)_{2 k^{2} k_{0}}} \subset \mathscr{A} \text { and } \mathscr{A}_{U(1)_{2 k^{2} k_{0}}} \subset \mathscr{A}_{U(1)_{2 k_{0}}}
$$

Since the vacuum representations $H_{\mathscr{A}}$ and $H_{\mathscr{A}_{U(1)_{2 k_{0}}}}$ have the same decompositions with respect to $\mathscr{A}_{U(1)_{2 k^{2} k_{0}}}$, we can identify $H_{\mathscr{A}}$ and $H_{\mathscr{A}_{U(1) 2 k_{0}}}$ and so assume that $\mathscr{A}_{U(1)_{2 k^{2} k_{0}}}$ is a common conformal subnet of $\mathscr{A}$ and $\mathscr{A}_{U(1)_{2 k_{0}}}$ on the same Hilbert space. Now choose unitary charged intertwiners $\phi_{i_{0}} \in \mathscr{A}(I)$ and $\psi_{i_{0}} \in \mathscr{A}_{U(1)_{2 k_{0}}}(I)$ such that $\operatorname{Ad}_{\phi_{i_{0}}}$ and $\operatorname{Ad}_{\psi_{i_{0}}}$ induce the same representation $\pi_{i_{0}}$ of $\mathscr{A}_{U(1)_{2 k^{2} k_{0}}}$. Define a unitary operator $U$ commuting with $\mathscr{A}$ such that $U \phi_{i_{0}}^{m} \Omega=\psi_{i_{0}}^{m} \Omega$ for all $m \in \mathbb{Z}$, where $\Omega$ is the vacuum vector. One checks easily that $U \mathscr{A}(I) U^{*}=\mathscr{A}_{U(1)_{2 k_{0}}}(I)$, $U \Omega=\Omega$. Since $U$ commutes with $\mathscr{A}$, and so it commutes with the action of $\operatorname{PSL}(2, \mathbb{R})$, we have $U \mathscr{A}(J) U^{*}=\mathscr{A}_{U(1)_{2 k_{0}}}(J)$ for all $J \in \mathscr{I}, U \Omega=\Omega$, thus proving that $\mathscr{A} \simeq \mathscr{A}_{U(1)_{2 k_{0}}}$.

The situation when $\mathscr{A}_{c=1} \subset \mathscr{A}_{\mathrm{SU}(2)_{1}}^{U(1)} \subset \mathscr{A}_{\text {is similar. } \mathscr{A}_{\mathrm{SU}(2)_{1}}^{U(1)} \text { has a continuous }}$ series of irreducible representations labeled by a real number $q$, which generate a fusion ring isomorphic to $\mathbb{R}$. By Lemma 4.3 and Corollary 3.9 we have $H_{\mathscr{A}}=$ $\bigoplus_{q \in S} H_{q}$ where $S \subset \mathbb{R}$ is an abelian subgroup, and $q^{2} \in 2 \mathbb{Z}$ for all $q \in S$. If $S=\{0\}$, then $\mathscr{A}=\mathscr{A}_{\mathrm{SU}(2)_{1}}^{U(1)}$. Assume that $S \neq\{0\}$, and let $q_{0}>0$ be the least positive number in the discrete set $S$. Then $S=\mathbb{Z} q_{0}$. Let $n$ be the positive integer such that $q_{0}=\sqrt{2 n}$; we have the decompositions $H_{\mathscr{A}}=\bigoplus_{k \in \mathbb{Z}} F_{\sqrt{2 n} k}$. Compare this with $\mathscr{A}_{\mathrm{SU}(2)_{1}}^{U(1)} \subset \mathscr{A}_{U(1)_{2 n} n}$, and an argument similar to the one above, using unitary charged intertwiners, shows that $\mathscr{A} \simeq \mathscr{A}_{U(1)_{2 n} \text {. }}$.

4.6.4. The case $\mathscr{A}_{c=1} \subset \mathscr{A}_{\mathrm{SU}(2),}^{D_{\infty}} \subset \mathscr{A}$. Let $O$ be the nontrivial one-dimensional representation of $D_{\infty}$ (the infinite Dihedral group). We will also use $O$ to denote the corresponding irreducible representation of $\mathscr{A}_{\mathrm{SU}(2)_{1}}^{D_{\infty}}$. We note that the conformal dimensions of $O$ are integers, and by [Rehren 1990], we can choose a representative of [O] such that $O^{2}=$ id. Also the braiding operator $\epsilon(O, O)$ is a scalar with property $\epsilon(O, O)^{2}=1$.

First consider the case when $\alpha_{O}$ is localized on $I$, i.e., $\alpha_{O}$ is a DHR representation of $\mathscr{A}$. Applying Doplicher-Roberts reconstruction to $\mathscr{A}$ and $\alpha_{O}$ as in [Müger 1999, Proposition 3.8], we get a conformal net $\mathscr{A}_{1}$ such that $\mathscr{A} \subset \mathscr{A}_{1}$ and

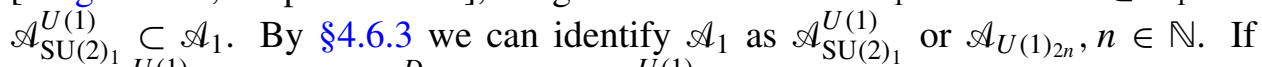
$\mathscr{A}_{1}=\mathscr{A}_{\mathrm{SU}(2)_{1}}^{U(1)}$, we have $\mathscr{A}_{\mathrm{SU}(2)_{1}}^{D_{\infty}} \subset A_{A} \subset \mathscr{A}_{\mathrm{SU}(2)_{1}}^{U(1)}$ and it follows that

$$
\mathscr{A} \simeq \mathscr{A}_{\mathrm{SU}(2)_{1}}^{D_{\infty}} \quad \text { or } \quad \mathscr{A} \simeq \mathscr{A}_{\mathrm{SU}(2)_{1}}^{U(1)},
$$


since $\mathscr{A}_{\mathrm{SU}(2)_{1}}^{D_{\infty}}$ is the fixed-point subnet under the $\mathbb{Z}_{2}$ action. If $\mathscr{A}_{1}=\mathscr{A}_{U(1)_{2 n} \text {, again }}$ $\mathscr{A}_{\mathrm{SU}(2)_{1}}^{D_{\infty}}$ is the fixed-point subnet of $\mathscr{A}_{U(1)_{2 n}}$ under the action of $D_{\infty}$, so $\mathscr{A}$ is the fixed-point net under the action of a closed subgroup of $D_{\infty}$ as in \$4.6.2. It follows that $\mathscr{A}$ is isomorphic to either $\mathscr{A}_{\mathrm{SU}(2)_{1}}^{D_{\infty}}, \mathscr{A}_{\mathrm{SU}(2)_{1}}^{U(1)}$ or $\mathscr{A}_{U(1)_{2 m}}$ for some $m \in \mathbb{N}$.

We now return to the general case. Consider the inclusions

$$
\mathscr{A}_{\mathrm{SU}(2)_{1}}^{D_{\infty}}(I) \subset \mathscr{A}(I) \subset \mathscr{A}_{\mathrm{SU}(2)_{1}}^{D_{\infty}}\left(I^{\prime}\right)^{\prime}
$$

on $H_{\mathscr{A}}$. Let $\gamma_{1}: \mathscr{A}_{\mathrm{SU}(2)_{1}}^{D_{\infty}}\left(I^{\prime}\right)^{\prime} \rightarrow \mathscr{A}(I)$ be the canonical endomorphism whose restriction to $\mathscr{A}(I)$ is the canonical endomorphism from $\mathscr{A}(I)$ to $\mathscr{A}_{\mathrm{SU}(2)_{1}}^{D_{\infty}}(I)$; see [Longo and Rehren 1995]. Let $v_{1} \in \operatorname{Hom}\left(\mathrm{id}, O_{\tilde{\epsilon}} O_{\epsilon}\right)_{\mathscr{A}_{\mathrm{SU}(2)_{1}}^{D \infty}(I)}$ be a unitary operator, and let $v_{O} \in \mathscr{A}_{\mathrm{SU}(2)_{1}}^{D_{\infty}}\left(I^{\prime}\right)^{\prime}$ be the unique unitary operator such that $\gamma_{1}\left(v_{O}\right)=v_{1}$. Notice that $\gamma\left(\tilde{\alpha}_{O} \alpha_{O}\right)=O_{\tilde{\epsilon}} O_{\epsilon} \gamma$, so $v_{O} a=\tilde{\alpha}_{O} \alpha_{O}(a) v_{O}$ for all $a \in \mathscr{A}(I)$. Restrict to $\mathscr{A}_{\mathrm{SU}(2)_{1}}^{D_{\infty}}(I)$ and recall that $O^{2}=\mathrm{id}$. Then $v_{O} \in \mathscr{A}_{\mathrm{SU}(2)_{1}}^{D_{\infty}}(I)^{\prime} \cap \mathscr{A}_{\mathrm{SU}(2)_{1}}^{D_{\infty}}\left(I^{\prime}\right)^{\prime}$, so $v_{O}$ commutes with $\operatorname{PSL}(2, \mathbb{R})$ by the strong additivity of $\mathscr{A}_{\mathrm{SU}(2)_{1}}^{D_{\infty}}$. On the other hand, Proposition 3.5(2) shows that $\tilde{\alpha}_{O} \alpha_{O}=\alpha_{O} \tilde{\alpha}_{O}$ since $\epsilon(O, O)$ is a scalar, and so $v_{O}^{2} \in \mathscr{A}_{\mathrm{SU}(2)_{1}}^{D_{\infty}}(I)^{\prime} \cap \mathscr{A}(I)=\mathbb{C}$. Multiplying by a scalar if necessary, we can assume that $v_{O}^{2}=$ id and $v_{O}^{*}=v_{O}$. Hence the subnet defined by $\hat{A}(J):=\mathscr{A}(J) \cap\left\{v_{O}\right\}^{\prime}$ is a conformal subnet of $\mathscr{A}$ and $\mathscr{A}_{\mathrm{SU}(2)_{1}}^{U(1)} \subset \hat{A}$ is also a conformal subnet. Now consider

$$
\beta_{O}:=\tilde{\alpha}_{O}^{A_{\mathrm{SU}(2) 1}^{U(1)} \rightarrow \hat{A}^{A}} \alpha_{O}^{\mathcal{A U ( 1 )}(2)} \rightarrow \hat{A} .
$$

By definition we have $v_{O} a=\beta_{O}(a) v_{O}$ for all $a \in \hat{A}(I)$, and by the definition of $\hat{A}$ we get $v_{O} a=a v_{O}=\beta_{O}(a) v_{O}$ for all $a \in \hat{A}(I)$, so $\beta_{O}=$ id. It follows that

$$
\left[\tilde{\alpha}_{O}^{\nexists U_{\mathrm{SU}(2)}^{U(1)} \rightarrow \hat{A}}\right]=\left[\alpha_{O}^{\nexists_{\mathrm{SU}(2)}^{U(1)} \rightarrow \hat{A}}\right]
$$

so $\alpha_{O}^{\mathscr{A}_{\mathrm{SU}(2)}^{U(1)} \rightarrow \hat{A}}$ is localized on $I$. Therefore we can apply the first part of this section to identify $\hat{A}$ as either $\mathscr{A}_{\mathrm{SU}(2)_{1}}^{D_{\infty}}, \mathscr{A}_{\mathrm{SU}(2)_{1}}^{U(1)}, \mathscr{A}_{U(1)_{2 m}}^{\mathbb{Z}_{2}}$ or $\mathscr{A}_{U(1)_{2 m}}$ for some positive integer $m$. Since $\hat{A} \subset \mathscr{A}$, to identify $\mathscr{A}$ it is enough to consider the case when $\hat{A} \simeq \mathscr{A}_{\mathrm{SU}(2)_{1}}^{D_{\infty}}$ or $\hat{A} \simeq \mathscr{A}_{U_{(1)_{2 m}}^{\mathbb{Z}_{2}}}$ since the other cases have been treated in $\S 4.6 .3$. We show that in this case $\hat{A}=\mathscr{A}$. Note that by definition the index of $\hat{A}(I) \subset \mathscr{A}$ is at most 2. So we just have to show that the index is not 2. Consider the inclusion $\hat{A}(I) \subset \mathscr{A}$. If the index is 2 , one checks easily that $\alpha_{O}$ is not localized on $I$ and $\left[\gamma_{\mathscr{A}}\right]=\left[1+\tilde{\alpha}_{O} \alpha_{O}\right]$

Let $f$ be the vector representation of $\hat{A}(I)$. Note that $[O f]=[f]$. It is now easy to check that

$$
\left\langle\alpha_{f}, \alpha_{f}\right\rangle=1,\left\langle\tilde{\alpha}_{f} \alpha_{f}, 1\right\rangle \geq 1
$$

It follows that $\alpha_{f}$ is localized on $I$, and so $\alpha_{O} \prec \alpha_{f}^{2}$ is also localized on $I$, a contradiction. 
4.6.5. The case $\mathscr{A}_{c=1} \subset \mathscr{A}_{\mathrm{SU}(2)_{1}}^{G} \subset A_{A}$ for $G=E_{6}, E_{7}, E_{8}$. Since $\mathscr{A}_{\mathrm{SU}(2)_{1}}^{G}$ is completely rational, so is $\mathscr{A}$, by [Kawahigashi and Longo 2004, Proposition 2.3]. If $\mathscr{A} \subset$ $\mathscr{A}_{1}$ is a conformal subnet of $\mathscr{A}_{1}$ and is irreducible, meaning that $\mathscr{A}(I)^{\prime} \cap \mathscr{A}_{1}(I)=\mathbb{C}$, then by the same result $\mathscr{A}_{1}$ is also completely rational, and $\mu_{\mathscr{A}_{1}} \leq \mu_{\mathscr{A}}$, with equality if and only if $\mathscr{A}=\mathscr{A}_{1}$ by Proposition 2.3. If $\mathscr{A} \subset \mathscr{A}_{1} \subset \mathscr{A}_{2}$ are conformal subnets with $\mathscr{A} \subset \mathscr{A}_{1}$ and $\mathscr{A} \subset \mathscr{A}_{2}$ irreducible, we see, again by [Kawahigashi and Longo 2004, Proposition 2.3], that $\mathscr{A} \subset \mathscr{A}_{2}$ has finite index, so $\mathscr{A} \subset \mathscr{A}_{2}$ is also irreducible [Longo 2003, Lemma 14].

We claim that among the irreducible conformal extensions of $\mathscr{A}$ there is an $\mathscr{A}_{\text {max }}$ that cannot be enlarged (that is, if $\mathscr{A}_{\max } \subset \mathscr{B}$ is an irreducible conformal subnet, then $\left.\mathscr{A}_{\max }=\mathscr{B}\right)$. Otherwise there would be an infinite sequence $\mathscr{A}_{\subset} \subset \mathscr{A}_{1} \subset \mathscr{A}_{2} \subset \ldots$ with distinct members and each pair $\mathscr{A}_{i} \subset \mathscr{A}_{i+1}$ irreducible. By Jones's theorem [1983] the index $\left[\mathscr{A}_{i+1}, \mathscr{A}_{i}\right]$ is at least 2 , and it follows from Proposition 2.3 that $1 \leq \mu_{\mathscr{A}_{i}} \leq 4^{-i} \mu_{\mathscr{A}}$ for all $i$, contradicting the fact that $\mu_{\mathscr{A}}<\infty$.

Let $\mathscr{A}_{\text {max }}$ be a maximal conformal extension of $\mathscr{A}$, so $\mathscr{A}_{\mathrm{SU}(2)_{1}}^{G} \subset \mathscr{A} \subset \mathscr{A}_{\max }$. Let $\mathscr{B}$ be the conformal subnet of $\mathscr{A}_{\text {max }}$ generated by $\mathscr{A}_{c=1}$ and the charged intertwiners associated with the set $\left\{L\left(1, p^{2}\right) \mid p \in \mathbb{Z}\right\}$. Note that $\mathscr{A}_{\mathrm{SU}(2)_{1}}^{G} \subset \mathscr{B}$. By $\S 4.6 .2$, we can identify $\mathscr{B}$ with $\mathscr{A}_{\mathrm{SU}(2)_{1}}^{G^{\prime}}$, where $G^{\prime}$ a closed A-D-E subgroup of $\mathrm{SO}(3)$. If $G^{\prime}$ is of type $D$, using $\S 4.6 .4$ to identify all possible $\mathscr{A}_{\text {max }}$, we see that $\mathscr{A}_{\text {max }}$ can be further extended, contradicting the maximality of $\mathscr{A}_{\max }$.

If $G^{\prime}$ is of type $E$, let $\hat{G}^{\prime}$ be the twofold covering group of $G^{\prime}$ in $\mathrm{SU}(2)$. For any irreducible representation $\lambda$ of $\hat{G}^{\prime}$, we use the same $\lambda$ to label the covariant representation of $\mathscr{A}_{\mathrm{SU}(2)_{1} G^{\prime}}$. The statistical dimensions of $\lambda$ are given by the PerronFrobenius eigenvectors labeled in [Goodman et al. 1989]. Consider the induction for the pair $\mathscr{B}=\mathscr{A}_{\mathrm{SU}(2)_{1}}^{G^{\prime}} \subset \mathscr{A}_{\max }$. By Lemma 3.3(3) and the definition of $\mathscr{B}$, each $\alpha_{\lambda}$ is irreducible. Define the index sets $S:=\left\{\lambda \mid \lambda \in \operatorname{Irrep} G^{\prime},\left[\alpha_{\lambda}\right]=\left[\tilde{\alpha}_{\lambda}\right]\right\}$ and $\hat{S}:=\left\{\lambda \mid \lambda \in \operatorname{Irrep} \hat{G}^{\prime},\left[\alpha_{\lambda}\right]=\left[\tilde{\alpha}_{\lambda}\right]\right\}$. The set $S$ consists of representations of $A_{\max }$ which have permutation symmetry. By Doplicher-Roberts reconstruction, as in [Müger 1999, Proposition 3.9], we conclude there is an irreducible conformal extension of $\mathscr{A}_{\max }$ and it follows by the maximality of $\mathscr{A}_{\max }$ that $S=1$, where 1 is the trivial representation. Since $\hat{S}^{2} \subset S$, there is at most one $\lambda \in \hat{S}-$ Irrep $G^{\prime}$ such that $\lambda^{2}=1$. By inspecting the $E$ graph in [Goodman et al. 1989, p. 14], we conclude that $\hat{S}=\{1\}$, and so

$$
\left\langle\alpha_{\lambda} \tilde{\alpha}_{\lambda}, \alpha_{\mu} \tilde{\alpha}_{\mu}\right\rangle=\left\langle\alpha_{\lambda} \alpha_{\bar{\mu}}, \tilde{\alpha}_{\mu} \tilde{\alpha}_{\bar{\lambda}}\right\rangle=\left\langle\sum_{\delta \in \operatorname{Irrep}} N_{\hat{G}^{\prime}}^{\delta} \alpha_{\delta}, \sum_{\delta^{\prime} \in \operatorname{Irrep} \hat{G^{\prime}}} N_{\mu \bar{\lambda}}^{\delta^{\prime}} \tilde{\alpha}_{\delta^{\prime}}\right\rangle=\delta_{\lambda \mu} .
$$

On the other hand, $\left\langle\gamma_{\mathscr{A}}, \alpha_{\lambda} \tilde{\alpha}_{\lambda}\right\rangle \geq 1$ by Frobenius reciprocity and definitions, and we have $\gamma_{\mathscr{A}} \succ \sum_{\lambda \in \operatorname{Irrep} \hat{G}^{\prime}}\left[\alpha_{\lambda} \tilde{\alpha}_{\lambda}\right]$. This implies $\left[\mathscr{A}_{\max }: \mathscr{A}_{\mathrm{SU}(2)_{1}}^{G^{\prime}}\right]^{2} \geq\left|\hat{G}^{\prime}\right|^{2}=4|G|^{2}$, and by Proposition 2.3 we get a contradiction, namely 


$$
1 \leq \mu_{\mathscr{A}_{\max }}=\frac{2\left|G^{\prime}\right|^{2}}{\left[\mathscr{A}_{\max }: \mathscr{A}_{\mathrm{SU}(2)_{1}}^{G^{\prime}}\right]^{2}} \leq \frac{1}{2} .
$$

It follows that $G^{\prime}$ must be of type $A$, so $\mathscr{A}_{\text {max }}$ can be identified with $\mathscr{A}_{U(1)_{2 m}}$ for some positive integer, by $\S 4$.6.3. By the maximality of $\mathscr{A}_{\max }$, either $m=1$ or $m$ is square free, i.e., not divisible by $k^{2}$ for any $k>1$. In the later case the principal graph of $\alpha_{L(1,1 / 4)}^{\not A \rightarrow A_{\max }}$ is $A_{\infty,-\infty}$ by Lemma 4.4(3). But by Lemma 3.3(3), $\alpha_{L(1,1 / 4)}^{\not A \rightarrow A_{\max }}$ is also the induced endomorphism of the vector representation $f$ from $\mathscr{A}_{\mathrm{SU}(2)_{1}}^{G}$ to $\mathscr{A}_{\mathrm{max}}$, and it has finite depth since $\mathscr{A}_{\mathrm{SU}(2)_{1}}^{G}$ is completely rational. This contraction shows that $\mathscr{A}_{\text {max }}$ must be identified with $\mathscr{A}_{U(1)_{2}}=\mathscr{A}_{\mathrm{SU}(2)_{1}}$. Now we have inclusions

$$
\mathscr{A}_{c=1} \subset \mathscr{A} \subset \mathscr{A}_{\mathrm{SU}(2)_{1}}
$$

Since $\mathscr{A}_{c=1}$ is the fixed-point subnet of $\mathscr{A}_{\mathrm{SU}(2)_{1}}$ under the action of $\mathrm{SO}(3)$, it follows that $\mathscr{A}$ is the fixed point subnet of $\mathscr{A}_{\mathrm{SU}(2)}$ under the action of a closed subgroup of $\mathrm{SO}(3)$ as in the end of $\S 4.6 .2$.

Proof of Theorem 4.6. If $\mathscr{A}_{c=1}=\mathscr{A}$ there is nothing to prove. Assume that $\mathscr{A}_{c=1} \neq \mathscr{A}$. Let $\mathscr{A}_{f} \subset \mathscr{A}$ be the conformal subnet generated by charged intertwiners associated with the set $\left\{L\left(1, p^{2}\right): p \in \mathbb{Z}\right\}$, as in Corollary 3.9(3). By the spectrum condition the conformal subnet $\mathscr{A}_{f}$ is larger than $\mathscr{A}_{c=1}$, i.e., $\mathscr{A}_{c=1} \subset \mathscr{A}_{f}$ but $\mathscr{A}_{c=1} \neq \mathscr{A}_{f}$. By Corollary 3.9(3) $\mathscr{A}_{c=1} \subset \mathscr{A}_{f}$ is discrete, and by $\S 4.6 .2$ $\mathscr{A}_{f}$ can be identified as conformal net containing $\mathscr{A}_{\mathrm{SU}(2)_{1}}^{U(1)} \mathscr{A}_{\mathrm{SU}(2)_{1}}^{D_{\infty}}$ or $\mathscr{A}_{\mathrm{SU}(2)_{1}}^{G}$, for $G=E_{6}, E_{7}, E_{8}$, and the theorem follows from $\$ 4$.6.3-4.6.5.

If the spectrum condition is violated, the canonical endomorphism $\gamma$ has strange properties, and we have been able to rule out some cases. In general it is still an open question whether the spectrum condition is always satisfied.

We point out one application of Theorem 4.6. By a result from [Xu 1999], the net associated with the coset $\mathrm{SU}(2)_{4} \subset \mathrm{SU}(2)_{2} \times \mathrm{SU}(2)_{2}$ has 13 irreducible representations, and this net has central charge $c=1$. Using the known character formulas one can verify that the spectrum condition is satisfied in this case, so this net must be identified with an element in the list (4-1) by Theorem 4.6. From the coset fusion rules in [Xu 1999] one immediately identifies the coset with $\mathscr{A}_{U(1)_{12}}^{\mathbb{Z}_{2}}$. This was first pointed out to me by C. Dong during our discussions on the "fixed point resolution" problem about the cosets, of which $\mathrm{SU}(2)_{4} \subset \mathrm{SU}(2)_{2} \times \mathrm{SU}(2)_{2}$ is the first nontrivial example [Xu 1999].

Acknowledgment. I thank Professors Chongying Dong, Jürgen Fuchs, Christoph Schweigert, and Yasuyuki Kawahigashi for useful comments, and especially Prof. Roberto Longo for pointing out the article [Longo and Roberts 1997], which helps improve Proposition 3.4. 


\section{References}

[Böckenhauer and Evans 1998] J. Böckenhauer and D. E. Evans, "Modular invariants, graphs and $\alpha$ induction for nets of subfactors, I", Comm. Math. Phys. 197:2 (1998), 361-386. MR 2000c:46121 Zbl 0924.46047

[Böckenhauer and Evans 1999a] J. Böckenhauer and D. E. Evans, "Modular invariants, graphs and $\alpha$ induction for nets of subfactors, III", Comm. Math. Phys. 205:1 (1999), 183-228. MR 2000j:46118 Zbl 0949.46030

[Böckenhauer and Evans 1999b] J. Böckenhauer and D. E. Evans, "Modular invariants, graphs and $\alpha$-induction for nets of subfactors, II", Comm. Math. Phys. 200:1 (1999), 57-103. MR 2000c:46122 Zbl 01271630

[Buchholz and Schulz-Mirbach 1990] D. Buchholz and H. Schulz-Mirbach, "Haag duality in conformal quantum field theory", Rev. Math. Phys. 2:1 (1990), 105-125. MR 92a:81106 Zbl 0748.46040

[Buchholz et al. 1988] D. Buchholz, G. Mack, and I. Todorov, "The current algebra on the circle as a germ of local field theories", Nuclear Phys. B Proc. Suppl. 5B (1988), 20-56. MR 90h:81067 Zbl 0958.22500

[Carpi 1999] S. Carpi, "Classification of subsystems for the Haag-Kastler nets generated by $c=1$ chiral current algebras", Lett. Math. Phys. 47:4 (1999), 353-364. MR 2000h:81139 Zbl 0946.47055

[D’Antoni et al. 2001] C. D’Antoni, R. Longo, and F. Rădulescu, "Conformal nets, maximal temperature and models from free probability”, J. Operator Theory 45:1 (2001), 195-208. MR 2002d:46054 Zbl 0994.46022

[Dijkgraaf et al. 1989] R. Dijkgraaf, C. Vafa, E. Verlinde, and H. Verlinde, "The operator algebra of orbifold models", Comm. Math. Phys. 123:3 (1989), 485-526. MR 91c:81132 Zbl 0674.46051

[Dong and Griess 1998] C. Dong and R. L. Griess, Jr., "Rank one lattice type vertex operator algebras and their automorphism groups", J. Algebra 208:1 (1998), 262-275. MR 99h:17029 Zbl 0918.17023

[Doplicher and Roberts 1989] S. Doplicher and J. E. Roberts, "Endomorphisms of $C^{*}$-algebras, cross products and duality for compact groups", Ann. of Math. (2) 130:1 (1989), 75-119. MR 90j:46058 Zbl 0702.46044

[Doplicher and Roberts 1990] S. Doplicher and J. E. Roberts, "Why there is a field algebra with a compact gauge group describing the superselection structure in particle physics", Comm. Math. Phys. 131:1 (1990), 51-107. MR 91k:81082 Zbl 0734.46042

[Fredenhagen and Jörß 1996] K. Fredenhagen and M. Jörß, "Conformal Haag-Kastler nets, pointlike localized fields and the existence of operator product expansions", Comm. Math. Phys. 176:3 (1996), 541-554. MR 97b:81066 Zbl 0853.46077

[Fuchs et al. 2002] J. Fuchs, I. Runkel, and C. Schweigert, "TFT construction of RCFT correlators, I: Partition functions", preprint, 2002. hep-th/0204148

[Gabbiani and Fröhlich 1993] F. Gabbiani and J. Fröhlich, "Operator algebras and conformal field theory”, Comm. Math. Phys. 155:3 (1993), 569-640. MR 94m:81090 Zbl 0801.46084

[Goodman and Wallach 1984] R. Goodman and N. R. Wallach, "Structure and unitary cocycle representations of loop groups and the group of diffeomorphisms of the circle", J. Reine Angew. Math. 347 (1984), 69-133. MR 86g:22024a Zbl 0514.22012

[Goodman et al. 1989] F. M. Goodman, P. de la Harpe, and V. F. R. Jones, Coxeter graphs and towers of algebras, Mathematical Sciences Research Institute Publications 14, Springer, New York, 1989. MR 91c:46082 Zbl 0698.46050 
[Guido and Longo 1992] D. Guido and R. Longo, "Relativistic invariance and charge conjugation in quantum field theory", Comm. Math. Phys. 148:3 (1992), 521-551. MR 93i:81121 Zbl 0771.46039

[Guido and Longo 1996] D. Guido and R. Longo, "The conformal spin and statistics theorem", Comm. Math. Phys. 181:1 (1996), 11-35. MR 98c:81121 Zbl 0858.46053

[Haagerup 1978] U. Haagerup, "On the dual weights for crossed products of von Neumann algebras, II: Application of operator-valued weights”, Math. Scand. 43:1 (1978), 119-140. MR 81e:46048b Zbl 0405.46053

[Izumi et al. 1998] M. Izumi, R. Longo, and S. Popa, "A Galois correspondence for compact groups of automorphisms of von Neumann algebras with a generalization to Kac algebras", J. Funct. Anal. 155:1 (1998), 25-63. MR 2000c:46117 Zbl 0915.46051

[Jones 1983] V. F. R. Jones, "Index for subfactors", Invent. Math. 72:1 (1983), 1-25. MR 84d:46097 Zbl 0508.46040

[Kawahigashi 2003] Y. Kawahigashi, "Classification of operator algebraic conformal field theories”, pp. 183-193 in Advances in quantum dynamics (South Hadley, MA, 2002), edited by G. L. Price et al., Contemp. Math. 335, Amer. Math. Soc., Providence, RI, 2003. MR 2004m:81144 Zbl 1053.81040

[Kawahigashi and Longo 2004] Y. Kawahigashi and R. Longo, "Classification of local conformal nets: Case $c<1$ ”, Ann. of Math. (2) 160:2 (2004), 493-522. MR 2123931 Zbl 02157795

[Kawahigashi et al. 2001] Y. Kawahigashi, R. Longo, and M. Müger, "Multi-interval subfactors and modularity of representations in conformal field theory", Comm. Math. Phys. 219:3 (2001), 631-669. MR 2002g:81059 Zbl 1016.81031

[Laredo 1997] V. T. Laredo, Fusion of positive energy representations of $\mathrm{LSpin}_{2 n}$, Ph.D. thesis, University of Cambridge, 1997.

[Longo 1989] R. Longo, "Index of subfactors and statistics of quantum fields, I", Comm. Math. Phys. 126:2 (1989), 217-247. MR 91c:46097 Zbl 0682.46045

[Longo 1990] R. Longo, "Index of subfactors and statistics of quantum fields, II: Correspondences, braid group statistics and Jones polynomial”, Comm. Math. Phys. 130:2 (1990), 285-309. MR 91j: 46083 Zbl 0705.46038

[Longo 1992] R. Longo, "Minimal index and braided subfactors", J. Funct. Anal. 109:1 (1992), 98-112. MR 93i:46109 Zbl 0798.46047

[Longo 1994] R. Longo, "A duality for Hopf algebras and for subfactors, I", Comm. Math. Phys. 159:1 (1994), 133-150. MR 95h:46097 Zbl 0802.46075

[Longo 2003] R. Longo, "Conformal subnets and intermediate subfactors", Comm. Math. Phys. 237:1-2 (2003), 7-30. MR 2004i:46091 Zbl 1042.46035

[Longo and Rehren 1995] R. Longo and K.-H. Rehren, "Nets of subfactors", Rev. Math. Phys. 7:4 (1995), 567-597. MR 96g:81151 Zbl 0836.46055

[Longo and Roberts 1997] R. Longo and J. E. Roberts, "A theory of dimension", K-Theory 11:2 (1997), 103-159. MR 98i:46065 Zbl 0874.18005

[Müger 1999] M. Müger, "On charged fields with group symmetry and degeneracies of Verlinde's matrix S”, Ann. Inst. Henri Poincaré Physique Théorique 71:4 (1999), 359-394. MR 2001b:81066 Zbl 0938.81018

[Petkova and Zuber 2002] V. B. Petkova and J.-B. Zuber, "Verlinde NIM-reps for charge conjugate $\mathrm{sl}(N)$ WZW theory", pp. 161-170 in Statistical field theories (Como, 2001), edited by A. Cappelli and G. Mussardo, NATO Sci. Ser. II Math. Phys. Chem. 73, Kluwer Acad. Publ., Dordrecht, 2002. MR 2010999 Zbl 1045.82002 
[Pimsner and Popa 1986] M. Pimsner and S. Popa, "Entropy and index for subfactors", Ann. Sci. École Norm. Sup. (4) 19:1 (1986), 57-106. MR 87m:46120 Zbl 0646.46057

[Popa 1994] S. Popa, "Classification of amenable subfactors of type II", Acta Math. 172:2 (1994), 163-255. MR 95f:46105 Zbl 0853.46059

[Popa 1995] S. Popa, Classification of subfactors and their endomorphisms, CBMS Regional Conference Series in Mathematics 86, CBMS, Washington, and Amer. Math. Soc., Providence, 1995. MR 96d:46085 Zbl 0865.46044

[Pressley and Segal 1986] A. Pressley and G. Segal, Loop groups, Oxford University Press, New York, 1986. MR 88i:22049 Zbl 0618.22011

[Rehren 1990] K.-H. Rehren, "Braid group statistics and their superselection rules", pp. 333-355 in The algebraic theory of superselection sectors (Palermo, 1989), edited by D. Kastler, World Sci. Publishing, River Edge, NJ, 1990. MR 1147467

[Rehren 1994] K.-H. Rehren, “A new view of the Virasoro algebra”, Lett. Math. Phys. 30:2 (1994), 125-130. MR 95b:81194 Zbl 0789.17018

[Rehren and Tuneke 2000] K.-H. Rehren and H. R. Tuneke, "Fusion rules for the continuum sectors of the Virasoro algebra with $c=1$ ", Lett. Math. Phys. 53:4 (2000), 305-312. MR 2002g:81048 Zbl 0966.81025

[Strătilă 1981] Ș. Strătilă, Modular theory in operator algebras, Editura Academiei, Bucharest, 1981. MR 85g:46072 Zbl 0504.46043

[Wassermann 1998] A. Wassermann, "Operator algebras and conformal field theory, III: Fusion of positive energy representations of LSU $(N)$ using bounded operators", Invent. Math. 133:3 (1998), 467-538. MR 99j:81101 Zbl 0944.46059

[Xu 1998] F. Xu, "New braided endomorphisms from conformal inclusions", Comm. Math. Phys. 192:2 (1998), 349-403. MR 99b:81139 Zbl 0908.46044

[Xu 1999] F. Xu, "Algebraic coset conformal field theories, II", Publ. Res. Inst. Math. Sci. 35:5 (1999), 795-824. MR 2002a:81270 Zbl 0949.46038

[Xu 2000] F. Xu, "Jones-Wassermann subfactors for disconnected intervals", Commun. Contemp. Math. 2:3 (2000), 307-347. MR 2001f:46094 Zbl 0966.46043

[Xu 2001] F. Xu, "Algebraic orbifold conformal field theories", pp. 429-448 in Mathematical physics in mathematics and physics (Siena, 2000), edited by R. Longo, Fields Inst. Commun. 30, Amer. Math. Soc., Providence, RI, 2001. MR 2002k:81136 Zbl 1041.81082

[Xu 2005] F. Xu, "3-manifold invariants from cosets”, J. Knot Theory Ramifications 14:1 (2005), 21-90.

Received April 22, 2003. Revised October 3, 2004.

\section{FENG XU}

DEPARTMENT OF MATHEMATICS

UNIVERSITY OF CALIFORNIA AT RIVERSIDE

2208 SPROUL HALL

RIVERSIDE, CA 92521

and

\section{HEBEI Normal UNIVERSITY}

xufeng@math.ucr.edu 